\title{
Neutrino super beam based on a superconducting proton linac
}

\author{
E. Baussan, ${ }_{1}^{1}$ J. Bielski, ${ }^{2}$ C. Bobeth, ${ }^{1}$ E. Bouquerel,${ }^{1}$ O. Caretta, ${ }^{3}$ P. Cupial, ${ }^{4}$ T. Davenne, ${ }^{3}$ C. Densham,${ }^{3}$ \\ M. Dracos, ${ }_{1}^{1}$ M. Fitton, ${ }^{3}$ G. Gaudiot, ${ }^{1}$ M. Kozien, ${ }^{2}$ L. Lacny, ${ }^{2}$ B. Lepers, ${ }^{1}$ A. Longhin, ${ }^{5}$ P. Loveridge, ${ }^{3}$ \\ F. Osswald, ${ }^{1}$ P. Poussot, ${ }^{1}$ M. Rooney, ${ }^{3}$ B. Skoczen, ${ }^{2}$ B. Szybinski, ${ }^{2}$ A. Ustrzycka, ${ }^{2}$ N. Vassilopoulos, ${ }^{1}$ \\ D. Wilcox, ${ }^{3}$ A. Wroblewski, ${ }^{2}$ J. Wurtz, ${ }^{1}$ V. Zeter, ${ }^{1}$ and M. Zito ${ }^{5 *}$ \\ ${ }^{1} I P H C$, Université de Strasbourg, CNRS/IN2P3, F-67037 Strasbourg, France \\ ${ }^{2}$ Cracow University of Technology, Warszawska 24 St., 31-155 Krakow, Poland \\ ${ }^{3}$ STFC Rutherford Appleton Laboratory, OX11 OQX Didcot, United Kingdom \\ ${ }^{4} A G H$ University of Science and Technology, Krakow, Poland \\ ${ }^{5}$ Irfu, CEA-Saclay, 91191 Gif-sur-Yvette, France
}

(Received 5 December 2012; published 17 March 2014)

\begin{abstract}
We present a new design study of the neutrino Super Beam based on the Superconducting Proton Linac at CERN. This beam is aimed at megaton mass physics, a large water Cherenkov detector, proposed for the Laboratoire Souterrain de Modane in France, with a baseline of $130 \mathrm{~km}$. The aim of this proposed facility is to study $C P$ violation in the neutrino sector. In the study reported here, we have developed the conceptual design of the neutrino beam, especially the target and the magnetic focusing device. Indeed, this beam presents several unprecedented challenges, related to the high primary proton beam power (4 MW), the high repetition rate $(50 \mathrm{~Hz})$, and the low kinetic energy of the protons $(4.5 \mathrm{GeV})$. The design is completed by a study of all the main components of the system, starting from the transport system to guide the beam to the target up to the beam dump. This is the first complete study of a neutrino beam based on a pebble-bed target capable of standing the large heat deposition of MW class proton beams.
\end{abstract}

DOI: 10.1103/PhysRevSTAB.17.031001

PACS numbers: 29.25.Pj, 29.27.Eg

\section{INTRODUCTION}

The recent discovery of neutrino oscillations [1] implies that neutrinos have a nonzero mass and that the mass eigenstates are different from the flavor eigenstates. The two sets of states are related through the Pontecorvo-MakiNakagawa-Sakata (PMNS) neutrino mixing matrix, governed by three angles labeled $\theta_{12}, \theta_{23}$, and $\theta_{13}$, and a phase parameter $\delta_{C P}$. The latter, if nonzero, is responsible for a $C P$-violating phenomena and could be related to the matterantimatter asymmetry in the Universe. These far-reaching consequences have aroused considerable interest worldwide in the precision measurement of the PMNS matrix and, in particular, of the $\delta_{C P}$ phase. This study can be performed by measuring neutrino oscillation over a long baseline, typically $100 \mathrm{~km}$ or more, with a massive far detector.

The neutrino beam needed for this experiment can be produced using the conventional technique of impinging a proton beam on a target and focusing the pions produced by the proton interactions in the target using magnetic devices. The neutrinos originate by the decay in flight of these pions in a decay tunnel. The main difficulty of this scheme is that

\footnotetext{
Corresponding author. marco.zito@cea.fr

Published by the American Physical Society under the terms of the Creative Commons Attribution 3.0 License. Further distribution of this work must maintain attribution to the author $(s)$ and the published article's title, journal citation, and DOI.
}

a very intense proton beam is needed in order to measure $C P$-violation phenomena in a far detector. This poses unprecedented technological challenges on the target and the magnet focusing system. This type of neutrino beam based on a multi-MW proton beam is called a super beam and is today the preferred option for the next step of experimental studies of the PMNS matrix.

This article reports a study of the proposed neutrino Super Beam based on the Superconducting Proton Linac (SPL) [2] at CERN and aimed at Megaton Mass Physics (MEMPHYS) [3], a large water Cherenkov detector, in the Laboratoire Souterrain de Modane (Frejus, France), with a baseline of $130 \mathrm{~km}$. The aim of this project is to study $C P$ violation in the neutrino sector.

EUROnu was a design study within the European Commission Seventh Framework Program, Research Infrastructures. This design study has investigated three possible options for a future high intensity neutrino oscillation facility in Europe. The work was done by the EUROnu consortium, consisting of 15 partners and an additional 15 associate partners [4].

In the study reported here, we have developed the conceptual design of the neutrino beam, especially the target and the magnetic focusing device. Indeed, this beam presents several unprecedented challenges, like the high primary proton beam power (4 MW), the high repetition rate $(50 \mathrm{~Hz})$, and the relatively low kinetic energy of the protons $(4.5 \mathrm{GeV})$. The design is completed by a study of all the main components of the system, starting from the 
transport system to guide the beam to the target up to the beam dump.

The first studies of this facility [5-8] were performed assuming a $2.2 \mathrm{GeV}$ proton beam and a liquid mercury jet target associated with a single conic horn with a pulsed current of $300 \mathrm{kA}$. Later it was proposed [9] to supplement the system with an auxiliary horn (called reflector) enclosing concentrically the first horn and operated at $600 \mathrm{kA}$ in order to focus also pions produced at larger angles. This scheme was adopted in [10] and the horn shape reoptimized using the method described in [11]. Further, the decay tunnel was reoptimized using different primary beam energies from 2.2 up to $8 \mathrm{GeV}$. Based on the neutrino fluxes of [10] and an improved parametrization of the far detector, the physics performances of the project were presented in [12] assuming a $3.5 \mathrm{GeV}$ proton kinetic energy.

With respect to previous studies on this subject we propose a new design based on the use of four identical solid targets and four magnetic horns operated with a lower value of the pulsed current (300-350 kA). We present a first complete study of a novel target for a neutrino beam, a pebble-bed target composed of small titanium spheres, cooled by a transversal flow of helium. Such a setup simplifies the engineering complexity of the system avoiding difficult issues such as the containment of the mercury jet in a magnetic field free region, the challenge of a power supply operating at $600 \mathrm{kA}$, and the constraints related to mechanical stresses on the horn-reflector system induced by the high frequency current pulsing.

The report is organized in the following way. In the next section, we briefly present the overall system and a summary of the main parameters and dimensions. We then present the various components, the beam transport, and distribution system (Sec. III), the target station (Sec. IV), the target (Sec. V), and the horn (Sec. VI). Finally, the study of the activation and shielding of the system is presented in Sec. VII and the neutrino fluxes and physics performances in Sec. VIII. This report presents only a summary of the main results obtained in the course of this study. A more complete description can be found in [13].

\section{OVERVIEW OF THE FACILITY}

A schematic view of the facility is shown in Fig. 1. $\mathrm{H}^{-}$ions are accelerated in the SPL, then injected into the accumulator ring. The resulting proton bunches are separated into four beam lines in the switchyard and sent onto the four targets. Four horns focus the mesons, mostly pions, into the decay tunnel where they decay predominantly through $\pi^{+} \rightarrow \mu^{+} \nu_{\mu}$. The resulting neutrino beam is aimed towards the far detector, with a $0.6^{\circ}$ inclination angle.

The possible layout [14] of the Super Beam facility at CERN is shown in Fig. 2. This layout is fully compatible with the existing CERN facilities and has been developed by the CERN EUROnu group together with the CERN Civil Engineering Service.

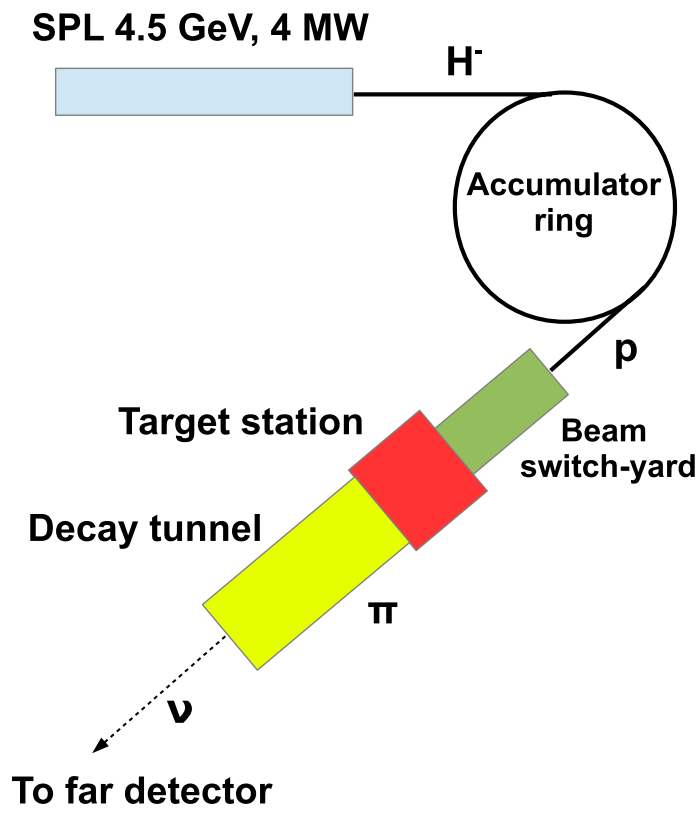

FIG. 1. Schematic view of the various components of the neutrino Super Beam.

The proton beam for this facility will be provided by the high power SPL, followed by an accumulator ring. To reduce the challenge on the target and the horn system, in particular, the heat to be removed, the stresses, and the radiation damage, we have foreseen a set of four identical target and horn units. Each target will then receive a full beam spill every $80 \mathrm{~ms}$ for a total power of $1 \mathrm{MW}$.

We present a view of the beam transport and distribution system in Fig. 3. The beam line, with a total length of $30 \mathrm{~m}$, is composed of two kickers, and then one dipole and three quadrupoles on each of the four separate transport lines.

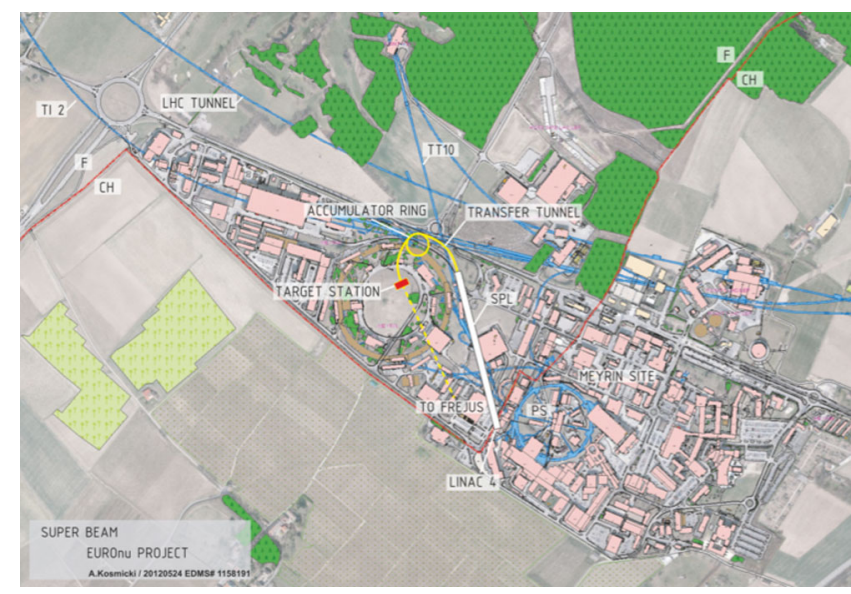

FIG. 2. Layout of the Super Beam facility at CERN. The yellow line shows the transfer lines from SPL and the accumulator ring, while the red box shows the location of the target station, in the ISR area. (Image provided courtesy of Elena Wildner and the CERN Civil Engineering Service.) 


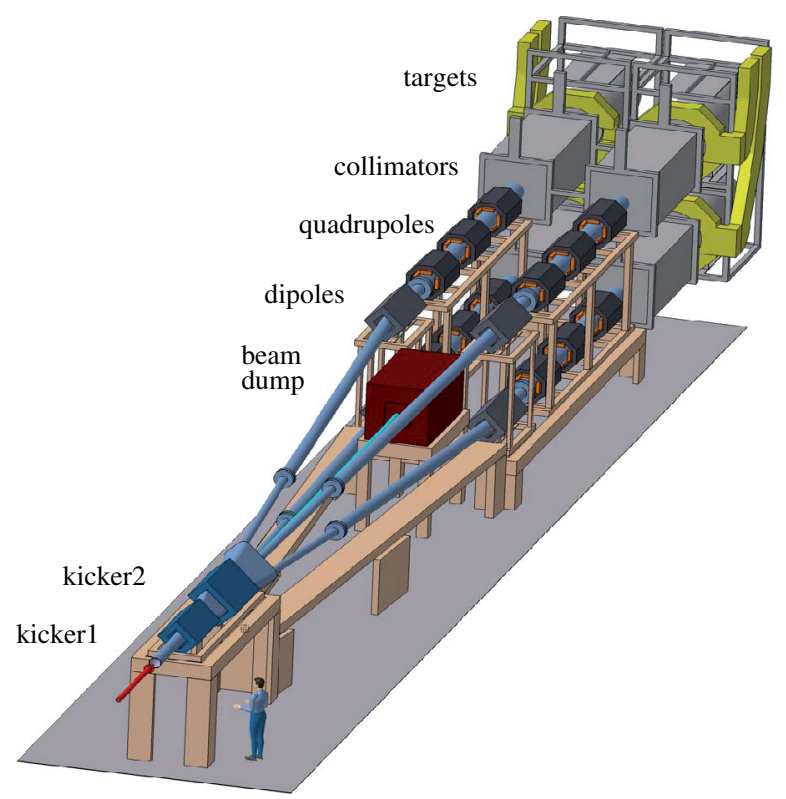

FIG. 3. The beam transport and distribution system.

The target station is shown in Fig. 4 and consists of the four targets and horns within a single large helium vessel. It is followed by the decay volume with a length of $25 \mathrm{~m}$ and by the beam dump. The thickness of the concrete shielding around the decay volume is $5.2 \mathrm{~m}$.

The target ( $78 \mathrm{~cm}$ long and $2.4 \mathrm{~cm}$ in diameter) is made out of a titanium can filled with a $3 \mathrm{~mm}$ diameter titanium sphere. It is cooled by a transversal helium flow. Each target is inserted inside a $2.5 \mathrm{~m}$ long magnetic horn, pulsed with a current of $350 \mathrm{kA}$.

\section{THE PROTON BEAM TRANSPORT AND DISTRIBUTION}

\section{A. The Superconducting Proton Linac}

The proton driver foreseen for this neutrino beam is the High Power Superconducting Proton Linac (HP-SPL) under study at CERN. The most recent design study [2] considers a beam power of $4 \mathrm{MW}$ at $50 \mathrm{~Hz}$ repetition frequency with protons of up to $5 \mathrm{GeV}$ kinetic energy and a pulse duration of about $600 \times \mu$ s for neutrino physics applications. The parameters considered for the SPL in the latest study are reported in Table I.

We have defined the baseline proton kinetic energy to be $4.5 \mathrm{GeV}$. This choice was based on a detailed study [15], taking into account the physics performances, the impact on the target, and the constraints from SPL. Larger kinetic energies are favored because the energy deposited in the target is lower, for a given beam power. This mitigates the technological difficulty of the target system. The SPL beam is limited to a maximum energy of $5 \mathrm{GeV}$. On the other hand, the physics performances do not depend strongly on the proton energy except for the kaon contamination that increases with energy. The neutrino flux, its composition, and the physics performances are discussed in Sec. VIII.

\section{B. The accumulator ring}

The pulses produced by the SPL have a duration of $0.6 \mathrm{~ms}$. However, the technology retained for focusing the pions with a magnetic horn (Sec. VI) is incompatible with this duration. Indeed the horn should withstand the severe heating produced by the current pulse (a $100 \mu \mathrm{s}$ semisinusoid pulse with a $350 \mathrm{kA}$ peak current) producing the magnetic field. A steady circulation of water in the cooling system of the horn allows one to reduce the temperature and the static stress. On the basis of these considerations we came to the requirement that the pulse duration of the proton beam delivered on the SPL-Super Beam target-horn station should be less than $5 \mu \mathrm{s}[16,17]$. This allows one to limit the electrical power sent to the horn system and thus keep the Joule effect to a reasonable level. For this reason an additional accumulator ring is required interfacing the SPL and the target-horn station. This section presents preliminary considerations on the accumulator.

Dedicated design studies have been performed for the Neutrino Factory $[18,19]$ that requires a combination of accumulator and compressor rings in order to achieve a

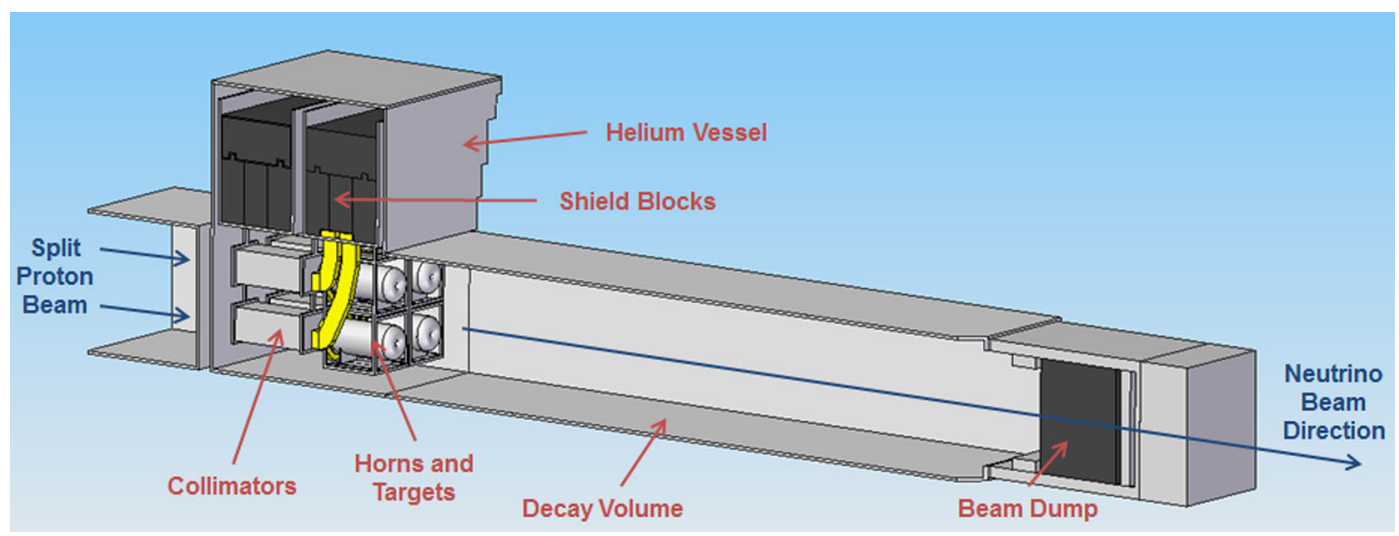

FIG. 4. Components of the target station, decay volume, and beam dump. 
TABLE I. Parameters of the HP-SPL [2].

\begin{tabular}{ll}
\hline \hline Parameters & Value \\
\hline Energy & $4.5 \mathrm{GeV}$ \\
Beam power & $4.0 \mathrm{MW}$ \\
Repetition rate & $50 \mathrm{~Hz}$ \\
Average pulse current & $40 \mathrm{~mA}$ \\
Transverse emittances (rms) & $0.45 \times \pi \cdot \mathrm{mm} \cdot \mathrm{mrad}$ \\
Beam width (rms) & $2 \mathrm{~mm}$ \\
Peak pulse current & $64 \mathrm{~mA}$ \\
Chopping ratio & $62 \%$ \\
Beam pulse length & $0.6 \mathrm{~ms}$ \\
Protons per pulse & $1.5 \times 10^{14}$ \\
Beam duty cycle & $2.0 \%$ \\
Number of klystrons (LEP, 352.2 MHz) & 14 \\
Number of klystrons (704 MHz) & 57 \\
Peak rf power & $219 \mathrm{MW}$ \\
Average power consumption & $38.5 \mathrm{MW}$ \\
Cryogenics avg. power consumption & $4.5 \mathrm{MW}$ \\
Cryogenic temperature & $2.0 \mathrm{~K}$ \\
Length & $534 \mathrm{~m}$ \\
\hline \hline
\end{tabular}

bunch length of $2 \mathrm{~ns}$ rms after compression. For the Super Beam the accumulator ring is sufficient and, among the considered scenarios, the 6-bunch per pulse option is the most favorable because of the lowest values of the local power distribution inside the target. This scenario foresees 6 bunches per pulse with bunch length $120 \mathrm{~ns}$ and gaps of $60 \mathrm{~ns}$. Because the original proton energy of the Neutrino Factory design was $5 \mathrm{GeV}$, the design will need to be finalized for the lower energy of $4.5 \mathrm{GeV}$ considered in this study.

\section{Beam distribution onto the horn system}

The incoming proton beam from the accumulator needs to be split into four different beams and impinged on the four target-horn system at a frequency of $12.5 \mathrm{~Hz}$. The general conceptual layout of the beam distribution is presented in Fig. 5.

The four targets are separated by a distance of $2000 \mathrm{~mm}$ (center to center). This value is a key parameter in the design of the beam distribution system as it determines the angle of deflection and thus the magnetic field mandatory for the splitting of the proton beam. The requirement on the Gaussian width of the beam is $4 \mathrm{~mm}$. The primary proton beam coming from the accumulator is assumed to propagate along the $z$ axis centered onto the 4-targets-horn system; two angles of deflection are therefore needed to bring the protons to the axis of each target. The use of two bipolar kickers would then be suitable to perform this task.

The two bipolar kickers $\mathrm{K} 1$ and $\mathrm{K} 2$ make an angle of $45^{\circ}$ with respect to the central beam axis. This rotation already introduces a first angle of deflection. Therefore, according to the polarity of the magnetic field of $\mathrm{K} 1$ (K2), the proton beam is distributed diagonally to the compensating dipoles D1 or D3 (D2 or D4), which deviate the beam to the corresponding target $\mathrm{T} 1$ or $\mathrm{T} 3$ (T2 or T4). To avoid unwanted optical phenomena such as chromaticity and to have the beam hitting the target with an incident angle of $90^{\circ}$, the optical system has to be symmetrical. Therefore, compensating dipoles (D1, D2, D3, and D4) are needed.

A scheme of the operation mode of each optical element involved in this configuration can be therefore suggested. A few ms before the protons enter the kicker system, the magnetic field of one of the two kickers increases to reach its maximum value. When getting between the magnets of the kicker, the protons are subject to the magnetic force induced and then are deflected by the angle $\pm \alpha$ to the corresponding compensating dipole. The repetition rate for the whole horn system is $50 \mathrm{~Hz}$, which gives a rate of $12.5 \mathrm{~Hz}$ for each of the targets. Having two kickers in a series implies the use of an adequate aperture of the second kicker in order to prevent the beam kicked from the first kicker to hit the magnet of the second one.

At a distance of $15 \mathrm{~m}$ and at a proton energy of $4.5 \mathrm{GeV}$, the kickers must induce a magnetic field of $0.8 \mathrm{~T}$ to deflect the beam to the axis of the compensating dipoles. The vertical aperture of the second kicker (K2) should be at least $60 \mathrm{~cm}$ to allow the beam to pass through without damaging the magnets. The code TRANSPORT [20] was used to estimate the size of the beam envelope between the kicker and the compensating dipole travelling through the four beam lines. The aim of the four beam lines is not only to distribute the proton beam to the horn system at a frequency of $12.5 \mathrm{~Hz}$ but also to deliver a beam having the optimum characteristics required for the pion production. The beam waist must be located in the middle of each of the targets (which are $78 \mathrm{~cm}$ long) and must have a regular Gaussian shape of width $4 \mathrm{~mm}$.

A beam abort dump will be located after the pair of bipolar kickers in order to stop the $4.5 \mathrm{GeV}$ energy proton beam in case of failure of the magnets. For a single pulse

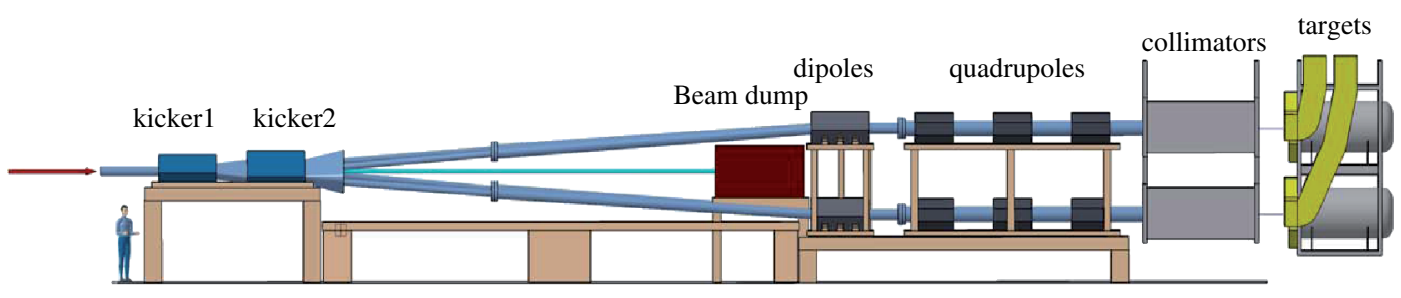

FIG. 5. Side view of the distribution system. 
TABLE II. Parameters of the accumulator [19].

\begin{tabular}{lc}
\hline \hline Parameters & Value \\
\hline Energy & $4.5 \mathrm{GeV}$ \\
Relativistic $\gamma$ & 6.32907 \\
Number of bunches & 6 \\
Beam width (rms) & $2 \mathrm{~mm}$ \\
Transverse emittances (rms) & $3 \times \pi \mathrm{mm} \mathrm{mrad}$ \\
Total bunch length & $120 \mathrm{~ns}$ \\
RMS momentum spread $(\mathrm{dp} / \mathrm{p})$ & $0.863 \times 10^{-3}$ \\
Circumference & $318.448 \mathrm{~m}$ \\
Average $\beta$ function $\left(\beta_{x}, \beta_{y}\right)$ & $(20,20) \mathrm{m}$ \\
Momentum compaction, $\alpha_{0}$ & 0.0249643 \\
Nominal tune, $Q_{x} / Q_{y}$ & $7.77,7.67$ \\
Natural chromaticity, $Q_{\text {nat }}^{\prime}$ & $-8.4,-7.9$ \\
Second order momentum compaction, $\alpha_{1}$ & 4.68 \\
Beam pipe half-height & $50 \mathrm{~mm}$ \\
\hline \hline
\end{tabular}

$\left(1.1 \times 10^{14}\right.$ protons $)$ the deposited energy in the dump represents $80 \mathrm{~kJ}$.

\section{Beam focusing}

To efficiently focus the beam onto the target system the use of optical elements such as quadrupoles is mandatory. Several configurations have been investigated with the code TRANSPORT including two or three quadrupoles.

The transverse size and the emittances of the proton beam entering the switchyard are considered to be similar to those of the beam leaving the SPL: $\sigma=2 \mathrm{~mm}$ and the rms emittances $\epsilon_{x}=\epsilon_{y}=3 \pi \mathrm{mmmrad}$ (Gaussian) (Table II). The relative errors on the emittances are considered to be $20 \%$ and are included in the simulations. A $1 \mathrm{~m}$ drift is considered between the entrance of the switchyard and the location of the first kicker. This is to allow space for a possible beam monitor to check the characteristics of the proton beam coming from the accumulator.

The baseline configuration of one beam line is presented in Fig. 6. The three quadrupoles (called QP1, QP2, and QP3) are here placed after the compensating dipole. The beam focuses at $29.9 \mathrm{~m}$, corresponding to the total length of the beam line plus the half length of the target, and its envelope at $3 \sigma$, satisfy the requirements (Fig. 6).

Table III summarizes the physical parameters calculated for the kickers, dipoles, and quadrupoles for each beam line of the switchyard system. Given the large magnetic field needed for the coils, the use of superconducting magnets will be investigated in further studies.

\section{E. Additional beam instrumentations}

During the experiment the quality and the position of the beam must be controlled at several positions along the beam lines and mainly at the entrance and the exit point of the switchyard system. Beam collimation may be needed upstream of the first kicker to cut off any eventual halo of the beam when leaving the accumulator. The exit point of the switchyard represents the interface with the target station and the last magnet. A large variation of the energy of the proton beam coming from the SPL accumulator may also induce chromatic focusing errors within the system. The addition of sextupoles may be required to correct this effect. Beam monitors should also be added at the exit point of the switchyard to measure the transverse position of the beam and make sure the beam hits the center of the targets as mentioned in the previous section. To suppress any eventual halo from the beam and to cope with beam fluctuations, one could consider a collimator at the exit point of the system.

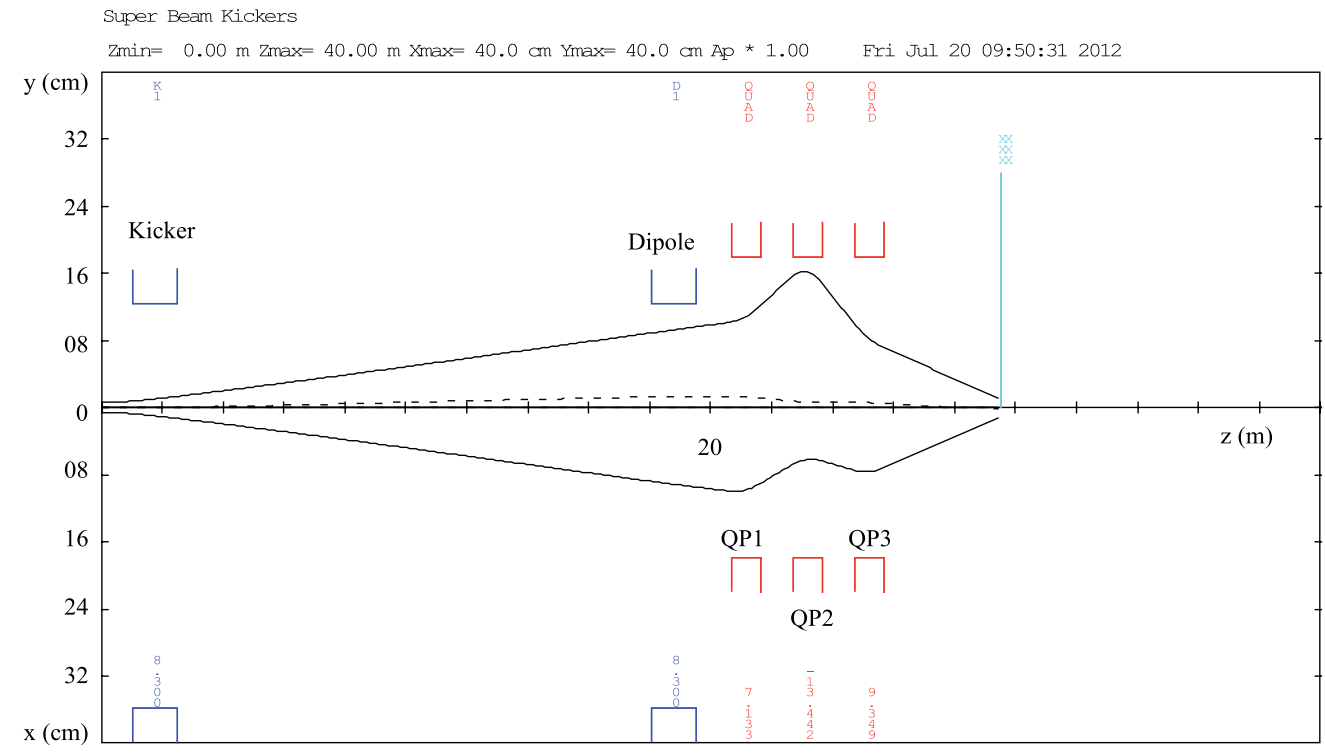

FIG. 6. Transverse beam envelopes. The dashed line represents the spatial dispersion. The initial beam parameters are $\left|x_{\max }\right|=\left|y_{\max }\right|=0.72 \mathrm{~cm} ;\left|x_{\max }^{\prime}\right|=\left|y_{\max }^{\prime}\right|=3.75 \mathrm{mrad}$; kinetic energy $4.5 \mathrm{GeV} ; d p / p=0.000863$. 
TABLE III. Summary of the physical parameters of: kicker 1,2 and dipole 1,2,3,4 (top), quadrupole 1,2,3 (bottom) of the 4 beam lines.

\begin{tabular}{lcccc}
\hline \hline & Kicker 1 & Dipole 1,3 & Kicker 2 & Dipole 2,4 \\
\hline Field strength $(\mathrm{T})$ & 0.83 & 0.83 & 0.96 & 0.96 \\
Angle of deflection (mrad) & \pm 83.0 & $\ldots$ & \pm 96.0 & $\ldots$ \\
Magnetic length $(\mathrm{m})$ & 1.5 & 1.5 & 1.5 & 1.5 \\
Aperture $H / V(\mathrm{~mm} / \mathrm{mm})$ & $250 / 350$ & $250 / 250$ & $250 / 600$ & $250 / 250$ \\
Total intensity $(\mathrm{kA})$ & 115.6 & 82.6 & 152.6 & 95.4 \\
\hline \hline & Quadrupole 1 & Quadrupole 2 & Quadrupole 3 \\
\hline Field gradient $(\mathrm{T} / \mathrm{m})$ & 0.71 & 1.34 & 0.93 \\
Aperture radius $(\mathrm{mm})$ & 180 & 180 & 180 \\
Magnetic length $(\mathrm{m})$ & 1 & 1 & $\mathrm{~F}$ \\
Function & $\mathrm{F}$ & $\mathrm{D}$ & 26.6 \\
Total intensity $(\mathrm{kA})$ & 20.3 & 38.4 & \\
\hline \hline
\end{tabular}

\section{F. The beam window}

The proton beam will be distributed equally among four targets, each of which will require its own beam window to separate the target station from the vacuum region of the four beam pipes. The main challenge is not to withstand the thermal stress induced by each pulse, but to remove the heat fast enough so that the window does not melt or fail by an accumulation of thermal deformation over many pulses.

Finite element analysis studies have concluded that beryllium windows, circumferentially cooled by forced convection water cooling, are a suitable design solution. The window should be thin (less than $0.5 \mathrm{~mm}$ ) to reduce the beam loss and have a partial hemisphere shape in order to withstand the differential pressure force between the target station and beam pipes. Simulations on a $0.25 \mathrm{~mm}$ thick window show that water cooling will be sufficient to keep the maximum temperature of the beryllium at the beam spot region below $200^{\circ} \mathrm{C}$. The peak stress is predicted to be less than one quarter of the yield stress of beryllium at the nominal operating temperature. The temperature and von Mises stress computed using ANSYS [21] for a $0.25 \mathrm{~mm}$ thick beryllium window circumferentially cooled by forced convection water (assuming a heat transfer of $2000 \mathrm{~W} / \mathrm{m}^{2} \mathrm{~K}$ ) are shown in Fig. 7.

The windows should be remotely replaceable and this could be achieved using inflatable bellowed seals on either side of the beryllium window. A similar design has already been employed successfully in the Japanese T2K neutrino facility [22].

\section{THE TARGET STATION}

\section{A. Introduction}

The target station contains sets of four baffle and collimators, targets, and magnetic horns within a single large helium vessel, along with the beam diagnostics and support infrastructure necessary for the safe and reliable operation of these components. The target station is separated from the primary beam line at the upstream end by four beam windows through which enters the split proton beam. The four split proton beams pass through the collimators, targets, magnetic horns, and decay volume, before being absorbed by the beam dump and hadron absorber.
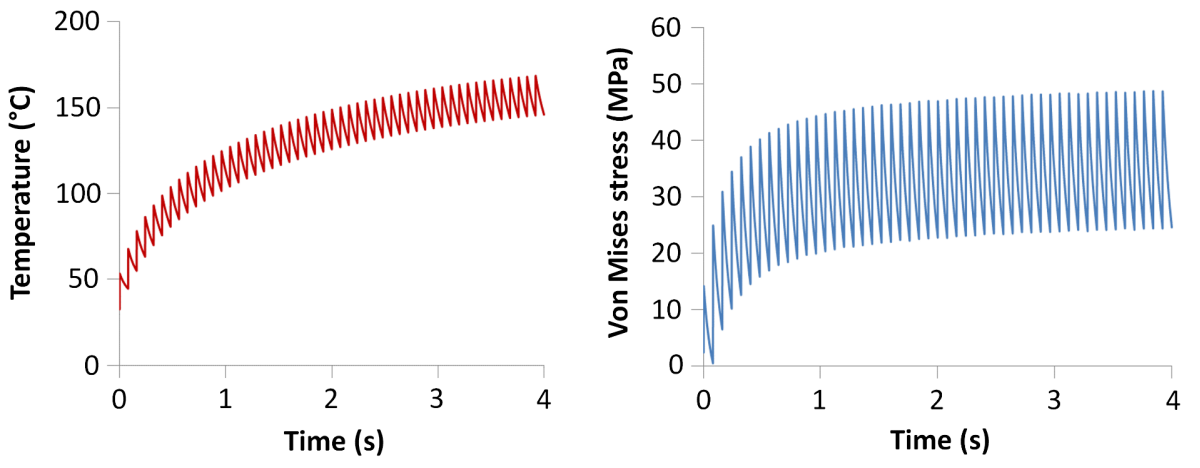

FIG. 7. Temperature (left) and von Mises stress (right) for a $0.25 \mathrm{~mm}$ thick beryllium window circumferentially cooled by forced convection water. 
The design of the target station must meet a number of important technical challenges. It requires substantial steel and concrete shielding. Because of the harsh conditions, horn and target lifetimes will be limited; multiple failures are expected during the lifetime of the facility. It is therefore essential that broken parts can be replaced, and due to the high activation this must be done using a remote handling system. Also, in order to generate a neutrino beam that is well aligned with the far detector, each target needs to be accurately positioned on the central axis of each magnetic horn. The use of four parallel horns will introduce further challenges unique to this facility. The cross section area of the beam line is increased by a factor of 4 , so a much larger volume of radiation shielding will be required to surround it. Having to accommodate four horns will increase the complexity of many operations, such as supporting the horns, connecting cooling and other services, replacing broken horns, and disposing of activated components.

The main objectives of the design process were as follows: (i) Ensure safe operation, and compliance with all applicable radiation limits. This includes ensuring the safety of repair workers and planning for the safe disposal of radioactive scrap. (ii) Minimize the amount of downtime required for repairs and maintenance. This will involve increasing the reliability of components and decreasing the time taken for repairs. (iii) Minimize the cost of construction, operation, and maintenance over the lifetime of the facility. The proposed design aims to deliver a compromise between reducing cost and reducing downtime.

The starting point for the design was the target station for the T2K experiment [22], located at the J-PARC facility in Japan. The T2K target station was designed to allow up to 4 MW beam power, with a remote handling system with similar capabilities to those required here. This is a proven design that has been running for 4 years (more than $6 \times 10^{20}$ protons on the first target), and is a valuable source of practical experience. Particular attention was paid in this new design to (i) reducing the time required to change a target or horn, and (ii) reducing the generation of tritium from the concrete within the helium volume.

For long-lived isotopes, tritium produced in water (from cooling several parts of the secondary beam line) will be diluted in water in several stages. For ${ }^{7} \mathrm{Be}$ and ${ }^{22} \mathrm{Na}$ ion exchangers will be used. The target station, decay tunnel, and beam dump will be in a closed-He environment as in T2K and opposite to air as in CERN Neutrinos to Gran Sasso (CNGS), for minimum activation (the production rate of ${ }^{3} \mathrm{H}$ in $\mathrm{He}$ is $1 / 25$ with respect to air). The amount of tritium generated in the $\mathrm{He}$ will be ventilated.

\section{B. Design overview}

The requirement for remote handling will be met by using an overhead gantry crane to insert and remove components from the beam line. All four horns will be mounted on a single support module that will provide support and alignment, and allow the horns to be lifted by the crane. The horns will then be moved to a maintenance area away from the beam for repair and disposal. This maintenance area will consist of the hot cell, where human operators can carry out repairs using remote manipulators, and the morgue, where activated scrap can be safely stored. In order to gain access to the components, the radiation shielding above them must first be removed. This will be achieved by making the top layer of shielding of movable concrete blocks that can be lifted off by the gantry crane. The beam line and maintenance area will be located at the bottom of a $10 \mathrm{~m}$ deep pit in order to prevent radiation shine to the outside when moving components. The target station vessel will be filled with helium at atmospheric pressure, in order to minimize pion absorption, tritium, and NOx production, and thus to provide an inert environment for the target and horns. The helium will be contained in a steel pressure vessel that will surround the horns, targets, collimators, and beam dump. Beam windows will separate the helium environment from the accelerator vacuum. The helium vessel will have a removable lid to allow access to the components inside.

In addition to the beam line and maintenance area, the target station must also contain the following systems: (i) cooling plant for the beam line components; (ii) power supply for the magnetic horns; (iii) air conditioning system for the buildings; (iv) pumps to fill and empty the helium vessel; (v) control room for the crane and other target station systems.

The proposed layout of the site is shown in Fig. 8. To reduce civil engineering costs, as many buildings as possible are located above ground. There are three surface buildings; a main hall containing the crane, horn assembly area, and access to the beam line, a side hall giving access to the horn power supplies and beam dump, and a pump house for cooling and air conditioning systems. The underground structure consists of a large pit beneath the main and side halls, which contains the beam line, maintenance area, and radiation shielding. The split proton beam enters the target station at a depth of $18 \mathrm{~m}$ and is angled by $0.6^{\circ}$ to point towards MEMPHYS.

\section{Helium vessel}

The horns, targets, collimators, decay volume, and beam dump will be contained in a steel vessel filled with helium at atmospheric pressure. Figure 9 shows the area covered by this vessel. Using helium will avoid the problems caused by passing a proton beam through air, such as the production of nitric acid which causes steel embrittlement, and the activation of large volumes of air. Using helium rather than vacuum will allow for cooling of components by conduction and convection, and will prevent stresses in the vessel due to differential pressure. However, the vessel will be required to temporarily withstand vacuum pressure as it will be vacuum pumped and 


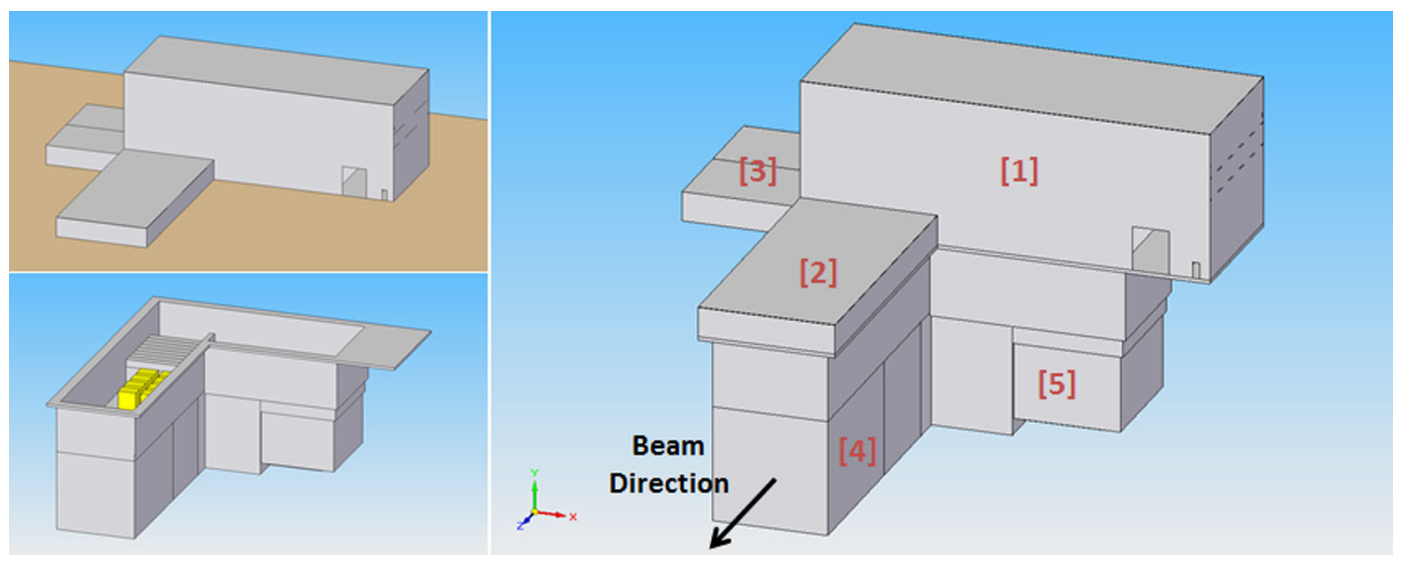

FIG. 8. Site layout: (1) main hall, (2) side hall, (3) pump house, (4) beam line shielding, and (5) maintenance area.

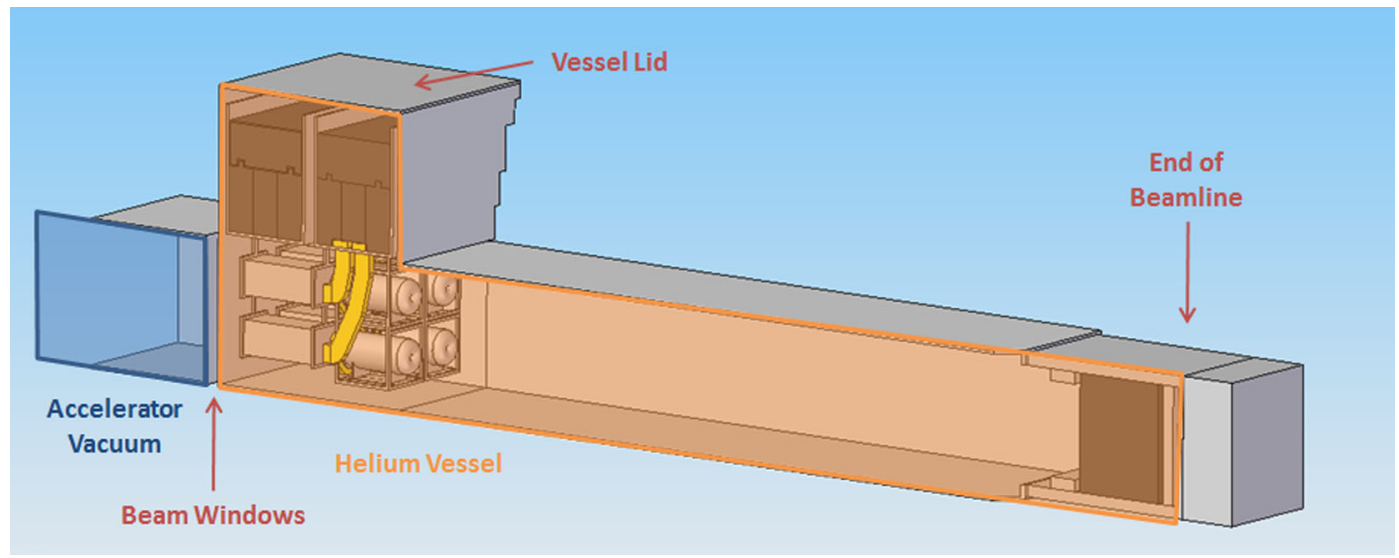

FIG. 9. Extent of helium vessel.

then backfilled with helium in order to achieve a high helium purity. The split proton beam will enter via four beam windows which will separate the helium vessel from the accelerator tunnel. The beam windows will connect to both sides via inflatable pillow seals, as used in T2K [22]. The benefit of pillow seals is that they can be remotely disconnected and do not depend on a mechanism to operate, so a damaged beam window can be replaced without requiring complex tooling or exposing a human repair worker to radiation. All four beam windows will be mounted on a single frame which can be lifted out by the gantry crane after the pillow seals have been disconnected. The hot cell will then be used to replace the damaged window without having to scrap the whole frame.

The helium vessel and the decay volume will be joined to form a single pressure vessel, as in $\mathrm{T} 2 \mathrm{~K}$. As a result, the entire decay volume must be pumped out every time the helium vessel needs to be opened in order to replace a component. Running the four targets at full power will cause a predicted heat load of $511 \mathrm{~kW}$ on the walls of the helium vessel and decay volume due to secondary particle interactions. As a result the walls will require active cooling, which will be achieved by using water channels on the outside of the vessel as for $\mathrm{T} 2 \mathrm{~K}$.

\section{Support module}

The horns and collimators will be held in place by support modules that can be lowered vertically into the helium vessel by crane, as shown in Fig. 10. One support module will hold the four horns, and a second will hold the four collimators. The support modules rest on kinematic mounts at the top of the helium vessel. Removable shield blocks will fit inside the support modules and rest on the sides of the vessel. The sides of the shield blocks will be stepped to create a labyrinth, preventing direct shine of radiation to the top of the vessel. The easiest place to disconnect services will be immediately after the feedthroughs, just inside the vessel. This will allow the connection points to be accessed from the top of the vessel without having to remove the shield blocks first. A mechanism for quickly disconnecting strip lines has been developed by Fermilab [23], and a similar design could be used here. 

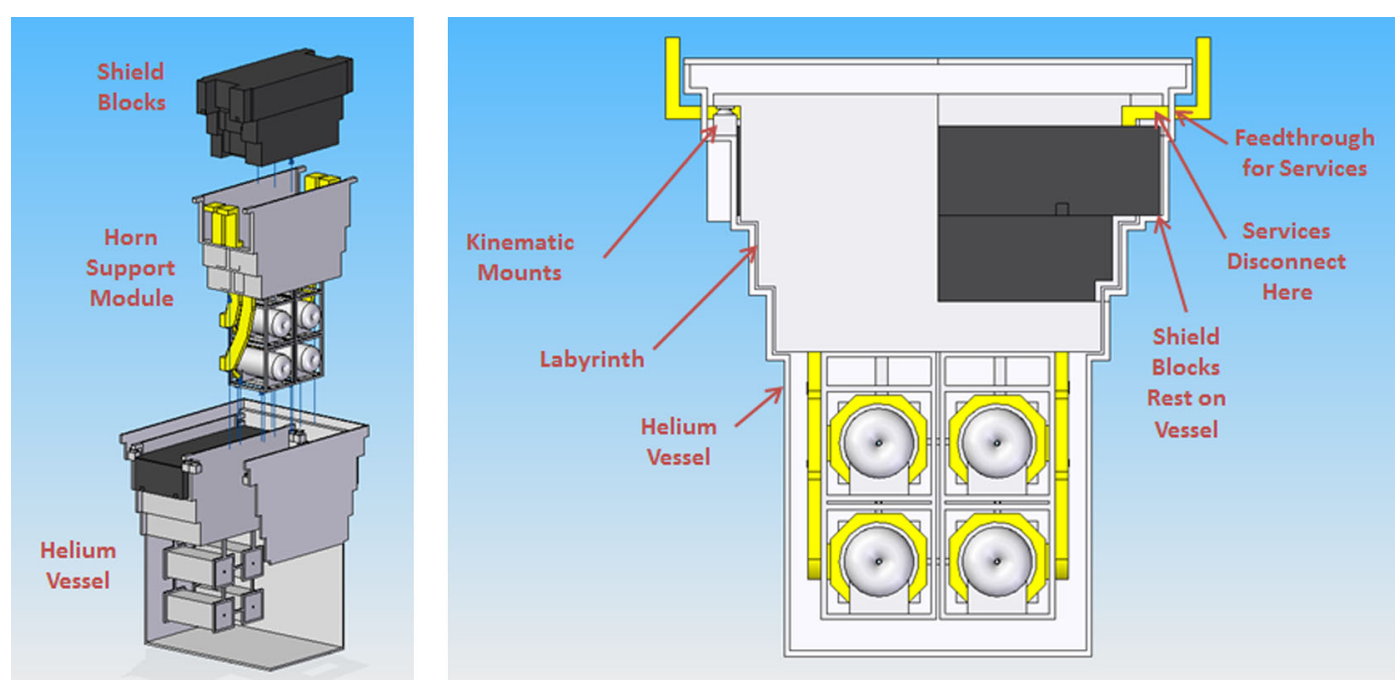

FIG. 10. Support modules, shield blocks, and helium vessel.

\section{E. Horn alignment}

It is essential that the four horns containing the four targets can be aligned with the four proton beams to submillimeter accuracy. This will depend on the alignment of the horns relative to the support module carrying them, and the alignment of the module relative to the helium vessel. This will be achieved by having the support modules rest on kinematic mounts, which are designed to exactly constrain the 6 degrees of freedom of motion. The kinematic mounts will allow the position of the module to be precisely defined in relation to the helium vessel, with high repeatability.

\section{F. Horn power supply}

Power for the magnetic horns will be provided by eight power supply units (PSUs) connected to the horns by strip lines. The length of strip line required should be reduced in order to minimize electrical losses. However, the PSUs must be located outside the radiation shielding to protect them from damage. This will be achieved by locating the PSUs on top of the decay volume shielding. This ensures that they are as close as possible to the horns. Above the beam dump shielding there will be space available for broken PSUs to be moved for maintenance. A 5 ton gantry crane in the side hall will be used to carry the PSUs.

The power supply is designed so that every horn must be connected to every PSU. The length of stripline must be roughly the same to each horn in order to ensure accurate timing, which is made more difficult by the fact that the lower horns will be further from the supply. Figure 11 shows the strip line layout that was designed to solve this. The length of strip line between the end of the PSUs and each horn is $20 \mathrm{~m}$, which is less than the specified maximum length. Each horn must be powered in turn as the beam is cycled around the four targets. Figure 11 shows which strip line connects to each horn, and also indicates the order in which the horns will be powered.

\section{G. Hot cell}

The hot cell will consist of a safe containment area for activated components and a shielded operator room. Repairs can be carried out by a human operator using remote manipulators to safely work on highly activated components. Two lead glass windows positioned at $90^{\circ}$ to each other provide direct visibility. The crane could be used to lift and rotate the component by $180^{\circ}$, in order to give a complete $360^{\circ}$ view. Access to the hot cell will be via a shaft from the control room building, allowing the hot cell to be accessed without having to enter the main hall. The roof of the hot cell will consist of removable concrete shield blocks, so it can be sealed when not in use. The roof of the operator room will also be made from shield blocks to allow for easy installation of manipulator arms using the main crane.

\section{H. Morgue}

The morgue will consist of a large underground space in which broken parts can be stored until their activation level has dropped enough that they can be moved elsewhere. Components in the morgue will be sealed in steel casks to stop most of the radiation. In addition to the casks, the morgue will be shielded by concrete walls on all sides. The morgue size specified here will have enough space to contain six complete support module assemblies, although one of these spaces will be filled by the spare horn assembly.

\section{Shielding}

The main source of radiation will be the horns and targets. To provide a biological shield, the helium vessel will be surrounded on all sides by a $2.2 \mathrm{~m}$ thick iron inner 


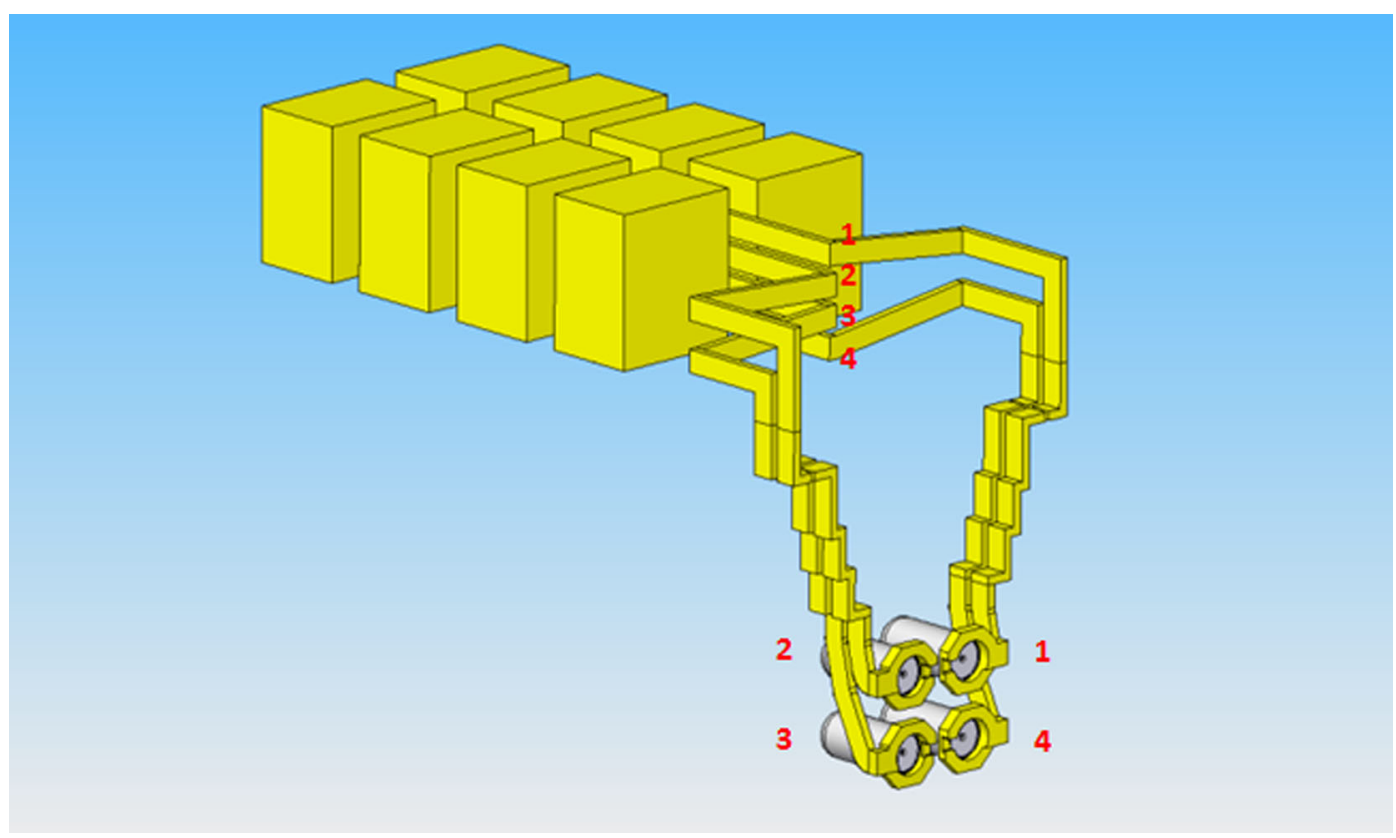

FIG. 11. Strip line connections from PSU to magnetic horns.

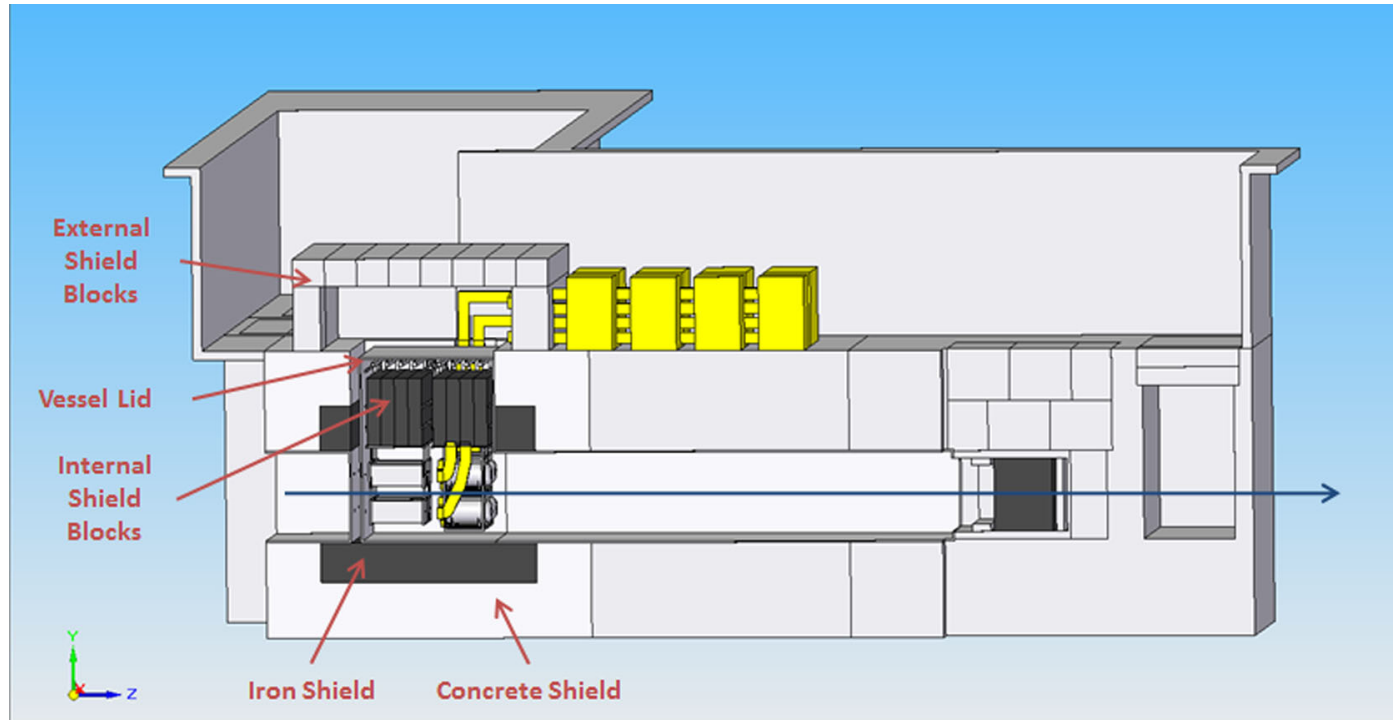

FIG. 12. Beam line shielding.

shield followed by a $3.7 \mathrm{~m}$ thick concrete outer shield. The rest of the beam line will be less active, and will be surrounded by a $5.2 \mathrm{~m}$ thick concrete shield. The maintenance area will also require shielding, around $2 \mathrm{~m}$ of concrete on all sides. Based on previous experience, it is recommended that low sodium concrete be used for the shielding, to limit the formation of radioactive sodium isotopes in the shielding. The outer concrete shield will need to be sealed to prevent activated air leaking from the region immediately surrounding the helium vessel into the target station atmosphere. There must be some way to open the shielding in order to gain access to the components inside. This will be achieved by making the top of each shield out of stacked concrete blocks that can be moved by the crane. Figure 12 shows the shielding arrangement around the beam line 7 and Fig. 13 shows the shielding of the helium vessel.

\section{J. Crane and control room}

Activated components will be moved using a 100 ton gantry crane. This crane will also be used for initial 

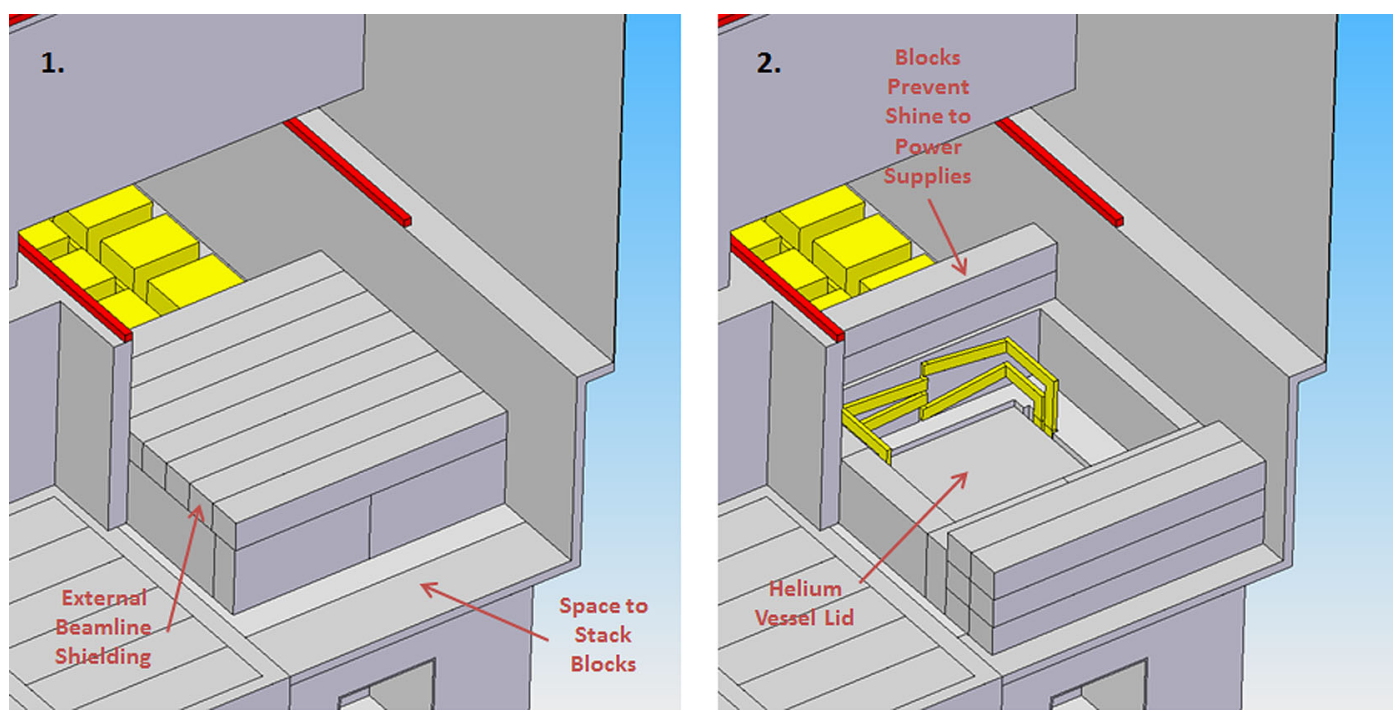

FIG. 13. External shielding of helium vessel: (1) closed and (2) open to access vessel.

installation of components and for moving replacement parts into the target station. There will also be a 5 ton gantry crane in the side hall, for carrying power supply units. The 5 ton and 100 ton cranes will overlap, so that the PSUs can be delivered to the main hall, unloaded by the large crane, then transferred to the small crane to be installed in the side hall.

\section{K. Maintenance procedure}

To minimize downtime, two assemblies of four horns each will be used at any one time. This will allow one assembly to be repaired while the other is running, so the beam will only have to be stopped for long enough to exchange the assemblies. The spare assembly will still be fairly active, and will therefore be stored in the morgue for safety. A procedure for a standard repair operation, for example replacing a broken target, has been considered.

\section{Decay volume}

The decay volume will consist of a $25 \mathrm{~m}$ long steel pressure vessel connecting the target station helium vessel to the beam dump. It will be directly connected to the helium vessel and so will also be filled with atmospheric pressure helium. The entire vessel will be built to withstand a vacuum when the helium is pumped out. The decay volume will be shielded with $5.2 \mathrm{~m}$ thick concrete on all sides. The steel vessel will experience significant heating from particle interactions and will therefore require its own cooling system. Downstream of the beam dump will be a pit to house muon monitors if required.

\section{Beam dump}

The beam dump will consist of graphite blocks, water cooled on two faces. The beam dump is designed to withstand normal $4 \mathrm{MW}$ operation, with the beam interacting in the targets. The incoming proton beam does not interact directly with the cooling water, in order to prevent water hammer and cavitation. To prevent graphite oxidation, the beam dump will be contained in the same helium vessel as the target station and decay volume. The graphite will be surrounded by iron plates, to reduce radiation dose to the surroundings. An upstream iron shield is designed to act as a collimator that will protect the cooling and diagnostic systems around the beam dump. Figure 14 shows the components of the beam dump.
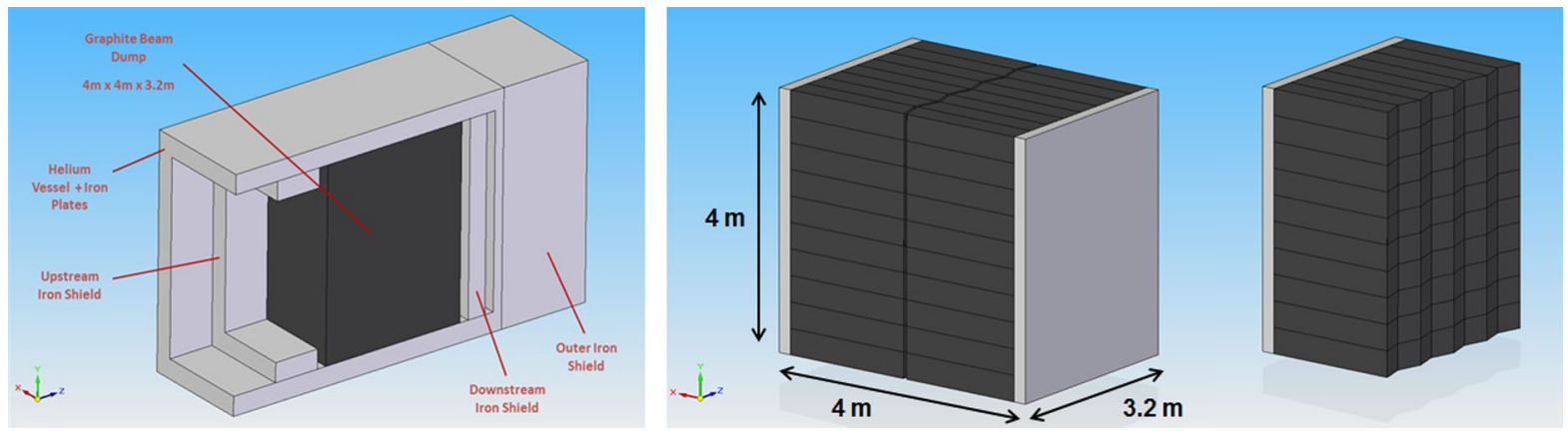

FIG. 14. Components of the beam dump (left) and graphite blocks (right). 

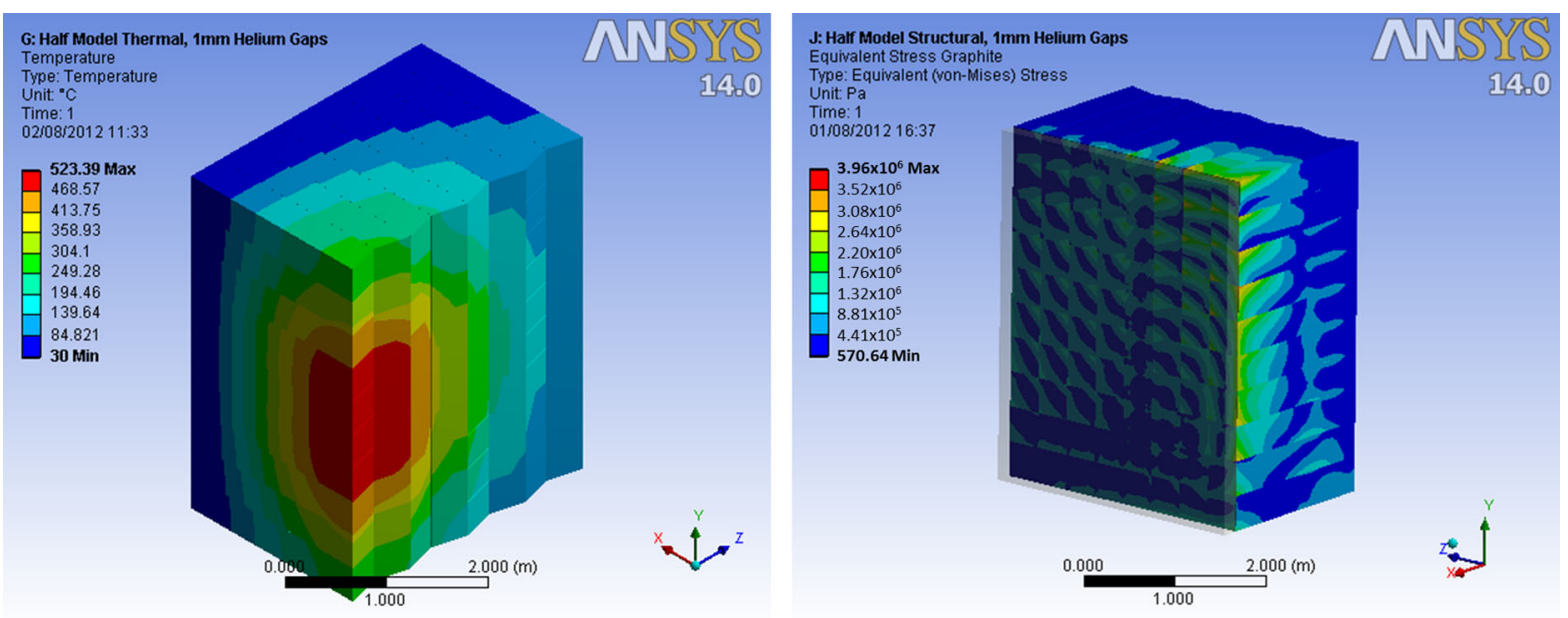

FIG. 15. Temperature (left) and von Mises stress (right) for the beam dump.

It is not possible to manufacture a single piece of graphite of the required size, so the beam dump must be built up from smaller blocks (Fig. 14). The end of each block will be cut at an angle, to prevent a direct shine path through the center of the beam dump. The grade of graphite used must be a trade-off between cost and material properties. The model presented here assumes Sec Carbon Ltd PSG-324 [24] is chosen-the same grade used for the T2K beam dump. This should be adequate, although a finer grade is recommended if affordable.

Simulations were carried out in ANSYS [21] to determine whether the proposed materials and design would be suitable. The heat load on the beam dump includes protons not absorbed by the target and undecayed pions. The beam dump is not designed to withstand the full, nondisrupted proton beam. In the event of beam miss-steer, the collimators will prevent damage to the beam dump. Simulations were carried out in ANSYS to model the beam dump performance under normal $4 \mathrm{MW}$ operation. The graphite temperature was modeled based on the following conservative assumptions: (1) all adjacent graphite blocks are separated by $1 \mathrm{~mm}$ gaps filled with helium; (2) heat transfer through the helium is by conduction only. The body temperature results were then used as input to a structural analysis. Temperature and stress results are presented in Fig. 15.

The rate of graphite oxidation increases with temperature, so the required purity of the helium environment will depend on the maximum temperature. Figure 15 shows a maximum temperature of $523{ }^{\circ} \mathrm{C}$. Based on the limits set out by $\mathrm{T} 2 \mathrm{~K}$ this means that the required helium purity will be better than $30 \mathrm{ppm} \mathrm{O}_{2}$, which should be feasible. The thermal performance of the proposed design should therefore be acceptable.

Figure 15 shows a maximum von Mises stress in the graphite of $3.96 \mathrm{MPa}$. This is fairly close to the tensile strength of the graphite (5 MPa). However, the majority of stress appears to be caused by the method of restraint rather than the actual thermal expansion. The proposed design should therefore be considered fit for use, as long as due consideration is given to the method of restraining the graphite.

\section{THE TARGET}

For the EUROnu Super Beam facility a high power target is required to generate pions to be focused by a magnetic horn. The target is expected to withstand the beam induced heating and associated stresses as well as offer reliable operation while exposed to intense radiation. The main technical challenges are as follows: (1) Heat removal. A significant heat load is deposited by the beam on the target and has to be removed reliably by the cooling system. (2) Static and dynamic stresses. The target must withstand thermal-mechanical stresses arising from the beam induced heating of the target. (3) Radiation damage. Degradation of the material properties due to radiation damage must be accommodated. (4) Geometrical constraints. The target has to fit inside the bore of the magnetic horn while having an appropriate geometry for effective pion production. (5) Remote replacement. Once activated the target has to be remotely manipulated in the event of failure. (6) Minimum expected lifetime. The target is expected to operate without intervention between scheduled maintenance shutdowns. (7) Safe operation. The target design should minimize any hazard to the personnel or the environment.

In the proposed concept, the target stands alone from the magnetic horn, has its own cooling system, and can be removed and replaced remotely. A combined target and horn design has also been considered but was rejected in favor of a separate target and horn system. The reasons for this decision are discussed in Sec. VA. Several target technologies have been considered and the most favorable concept is presented in the following sections. 


\section{A. Design philosophy}

Two outline target design concepts have been considered, namely, (1) a combined target and horn inner conductor, (2) a separate target and inner conductor, with the target supported within the horn bore.

Studies [25] have shown that the latter of these two options is preferable and this has been adopted as the baseline. A separate target and horn inner conductor was found to be preferable for the following reasons: (1) Removing the beam heating of the target and the Joule heating of the horn are both significant challenges. Separation of the two items reduces the challenge and permits separate cooling solutions. (2) More favorable target designs and cooling options, including segmented targetsm are possible, since the target is not required to conduct the horn current. A segmented target offers increased tolerance to accidental off-center beam conditions. (3) The thermal stress in the target is reduced without the additional Joule heating from the horn current pulse. (4) An increase in the horn inner conductor radius is possible, which significantly reduces the magnetic stress. (5) It becomes possible to tune the target and horn geometry separately, both radially and longitudinally, which permits a greater scope for optimization of the neutrino yield. (6) Failure modes are not combined, possibly leading to longer lifetimes for both target and horn. (7) Targets can be replaced separately within the horn, reducing cost of replacement and quantity of radioactive waste.

\section{B. Target cooling}

A $1 \mathrm{MW}$ proton beam with a kinetic energy of $4.5 \mathrm{GeV}$ deposits of the order of $50-85 \mathrm{~kW}$ of heat in the solid target for the materials that we have considered. Both contained water and helium gas cooling have been considered.

Helium cooling is preferred because there is negligible interaction between the beam and coolant making it readily possible for the coolant to be within the beam footprint for more direct cooling of the target. Beam-induced pressure waves in a gaseous coolant are largely reduced if compared with a liquid coolant, little activation of the helium is expected, and there are no corrosion issues with the target and cooling circuit materials. Several different target cooling geometry options are possible. Challenges or disadvantages of helium cooling compared with water include the fact that a relatively high pressure (larger than 10 bar) is required to generate a sufficient mass flow while limiting gas velocity and pressure drop to acceptable levels.

\section{Thermomechanical design of the target \\ 1. Packed bed of titanium spheres}

A packed bed of target spheres has been considered because of its inherent lower quasistatic and inertial dynamic stresses. This kind of arrangement for the target material was originally proposed in [26] in the context of the neutrino factory studies. However this is the first time that this target has been studied and modeled in detail, using finite element analysis software to assess quantitatively the target performance under the heat load. The packed bed target is made up of a canister containing many small target spheres. The packed bed canister has dimensions of the same order as the monolithic target but the individual spheres are much smaller. This has three advantages in terms of stress: (1) The spheres are almost uniformly heated by the beam because of their size and have a much shorter conduction path from the center of the sphere to the cooled surface. This means the temperature gradients in small spheres are small with respect to a larger monolith of the same thermal conductivity. The quasistatic stresses are driven by the temperature gradient and they are correspondingly lower. (2) The expansion time of a small sphere is much shorter than that of the solid monolith of the same material. In the case of the monolith the expansion time is longer than the pulse duration and as such significant inertial stresses occur. With small spheres the expansion time can be less than the pulse duration and so inertial stresses as a result of rapid energy deposition are negligible. (3) In the event of an off-center beam hitting a target, an asymmetric temperature profile is set up. This will have the effect of bending a solid monolith target and producing additional stress oscillations. As the spheres in a packed bed are not connected to each other and experience a close-to-uniform energy deposition whether the beam is on center or not, the packed bed configuration is inherently insensitive to an of- center beam.

Compared to the solid monolith target the packed bed has a lower density. Beryllium has been considered for the solid target that has a density of about $1.85 \mathrm{~g} / \mathrm{cc}$. The bulk density of the spheres cannot exceed $74 \%$ of the solid density. The density of the target material has an important effect on pion yield and so in order to recover the bulk density loss, titanium, which has a density of $4.5 \mathrm{~g} / \mathrm{cc}$, is proposed as a candidate material. A comparable pion yield from the surface of a solid beryllium target and a $74 \%$ density titanium target has been demonstrated using detailed simulation [27]. A titanium packed bed has been evaluated from a physics point of view with favorable results.

The packed bed canister would have a diameter just larger than the upstream baffle to protect it from a direct hit from the beam. It would be surrounded by coolant flow channels and would be perforated to allow the coolant to pass through the center of the target. This configuration gives rise to significantly more surface area for heat transfer than is present with the monolith target. The ideal flow configuration is transverse, i.e. the coolant passes through the packed bed in a direction perpendicular to the beam (Fig. 16). This minimizes pressure drop and so allows a greater volume flow through the target. As with all solid high power targets that are gas cooled, an advantage can be 


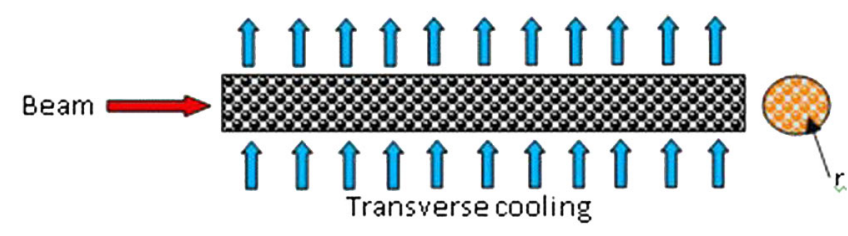

FIG. 16. Packed bed ideal flow configuration.

gained by pressurising the coolant. This allows an increase in mass flow without increasing the required pressure drop to drive the gas through the target. The larger surface-tovolume ratio with respect to the monolithic target and the proximity of the coolant to the core of the target offers potential for greater heat dissipation.

\section{Packed bed model}

An example case of a packed bed of Ti6Al4V spheres with transverse flow has been modeled. Energy deposition in the spheres has been calculated from a FLUKA model [28] of a titanium solid target with half density. Titanium has better thermal conductivity than its alloys but some alloys, such as Ti6Al4V, have much higher strength and as such Ti6A14V has been chosen for this example. Obtaining a practical transverse flow configuration within the confines of the magnetic horn is not trivial; however, a scheme is described here and some preliminary conjugate heat transfer modeling (using ANSYS CFX [29]) on an example case with a $1 \mathrm{MW}$ beam has been carried out. The geometry involves three inlet and three outlet channels spread at $120^{\circ}$ around the canister (Fig. 17). Holes of various sizes are strategically placed in the canister to allow gas to flow through the packed bed. The packed bed is modeled as a porous domain and appears to act as a diffuser with the flow naturally dividing evenly throughout the porous domain.
The pressure drop in the porous domain is calculated using the Ergun equation [30]

$$
\Delta P=\frac{f_{p} \rho V_{s}^{2}(1-\epsilon) L}{D_{p} \epsilon^{3}},
$$

where $f_{p}$ is a function of the packed bed Reynolds number, $\rho$ is the density of fluid, $V_{s}$ is the superficial velocity, $L$ is the length of the bed, $D_{p}$ is the equivalent spherical diameter of the packing, and $\epsilon$ is the void fraction of the bed.

A mass flow of helium of $93 \mathrm{~g} / \mathrm{s}$ is used with an outlet pressure of 10 bar. The pressure drop in the packed bed itself seems perfectly manageable and it appears as though there is scope for higher flow rates. Experience so far indicates that flow in the channels and, in particular, through the holes into the packed bed is the most significant cause of pressure drop. However, the predicted pressure drop of 1.1 bar appears reasonable and little design optimization has yet to be put into this example case. The maximum helium temperature is $584^{\circ} \mathrm{C}$ although the average outlet temperature is only $109^{\circ} \mathrm{C}$. This difference is due to the energy deposition in the packed bed not being uniform. The maximum sphere temperature is calculated to be $673^{\circ} \mathrm{C}$ (Fig. 17). The maximum steady state (ignoring temperature jump) sphere temperature, $T_{c}$, depends on the size of the sphere, $D_{p}$, conductivity of the sphere material, $k$, and the surface temperature, $T_{s}$,

$$
T_{c}-T_{s}=\frac{Q\left(D_{p} / 2\right)^{2}}{6 k},
$$

where $Q$ is the energy deposition $\left(\mathrm{W} / \mathrm{m}^{3}\right)$. The surface temperature depends on the heat transfer coefficient between the coolant gas and the sphere. This is calculated
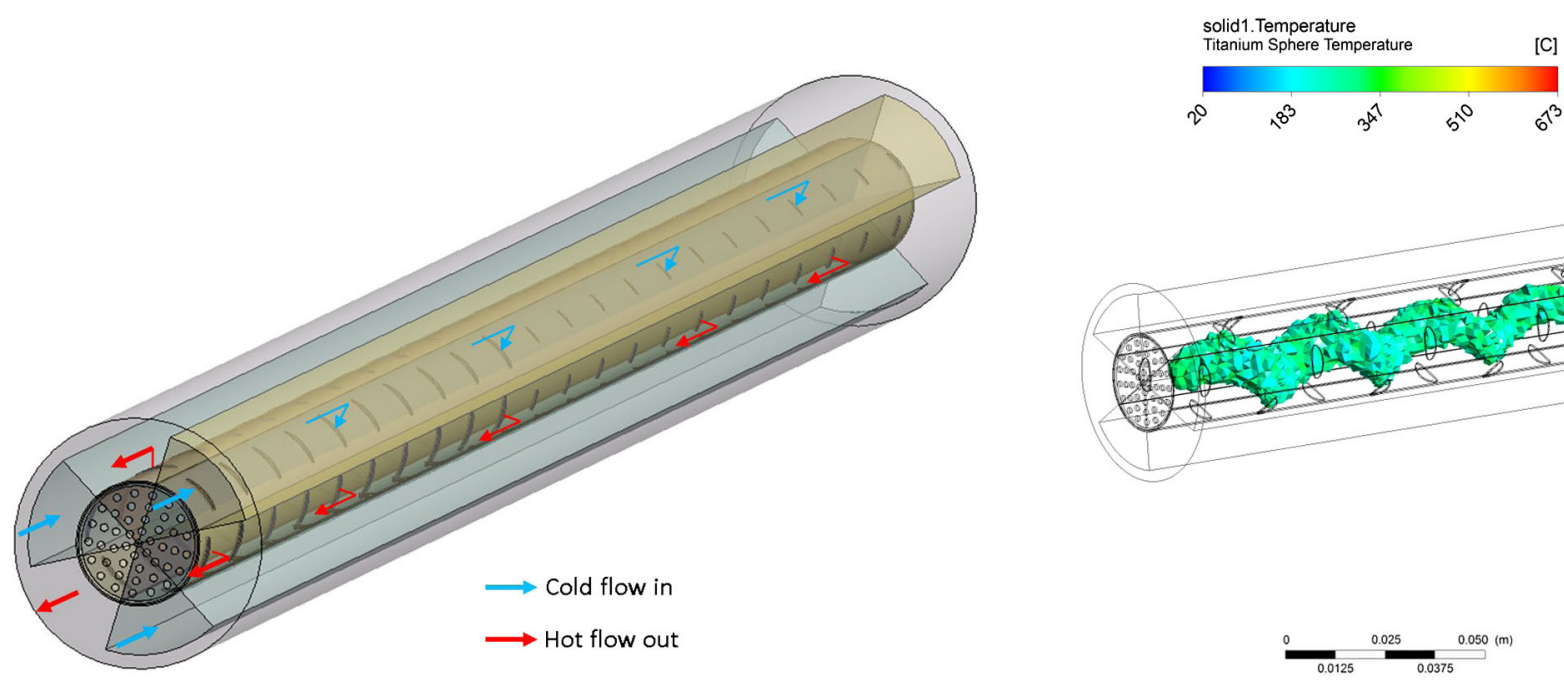

FIG. 17. Proposed coolant flow for the target (left) and temperature distribution (right). 
from a Nusselt number $N u$ correlation [31] for heat transfer in pebble beds with high Reynolds number $R e$

$$
N u=\left[\left(1.18 R e^{0.58}\right)^{4}+\left(0.23 R e^{0.75}\right)\right]^{0.25} .
$$

The three outlet channels are common and are configured such that the structure does not experience any significant asymmetries in its temperature profile.

Below follows a list of the key areas that need further work for the development of the packed bed target concept.

(1) The requirement for pressurized cooling gas necessitates a beam window that can withstand the pressure difference between a vacuum and the coolant pressure.

(2) Slight movements between the packed spheres may occur as a result of the sudden temperature jumps and corresponding thermal expansion. The titanium spheres with the highest energy deposition will have a temperature jump of $83^{\circ} \mathrm{C}$ with a $1 \mathrm{MW}$ beam.

(3) If the beam pulse is much shorter than the expansion time of the spheres this could give rise to an additional shock stress (assuming instantaneous heating). However the expansion time of the spheres is very small (a fraction of a microsecond) with respect to a solid monolith target so these inertial stresses are likely to be less important. Nonetheless this should be checked.

(4) The canister would need to accommodate the thermal expansion of the target spheres.

(5) The beam must pass through the canister to enter the packed bed, and a perforated cooled plate is envisaged to enclose the spheres while allowing coolant to pass through so as to minimize temperature gradients in the perforated plate. Stress analysis of this component is required.

(6) Off-center beam effects on the packed bed canister should be evaluated.

\section{How much heat can be removed from a packed bed?}

The limiting factors for the heat dissipation capability of a packed bed are the coolant exhaust temperature, the coolant pressure drop across the target, and the peak temperature and stress in the target spheres. For this 1 MW example modeled here it appears there is some headroom in terms of the key limiting factors: a target capable of dissipating a multimegawatt beam may be possible. This has been claimed for the case of a high $Z$ packed bed [26]. In order to find the practical limit of a packed bed, some further analysis and computational fluid dynamics is required.

\section{THE HORN}

\section{A. Hadrons focusing system: The electromagnetic horn}

In the case of the CERN SPL Super Beam the operation conditions of the horn will be much more severe than in previous applications. The proton driver power (4 MW) and repetition rate $(50 \mathrm{~Hz})$ are considerably higher than for previous experiments. The main failures observed in the past horns were related to the ancillary parts (cooling pipes, etc.) and not the horns themselves. More information about other horns can be found in [32].

A first step to mitigate the problem has been taken by splitting the beam onto four identical targets and horns, as described previously. In the following we study the horn for this design option.

\section{B. Horn design}

An initial design of a horn prototype system [6,7] foreseen for a neutrino factory has been made at CERN

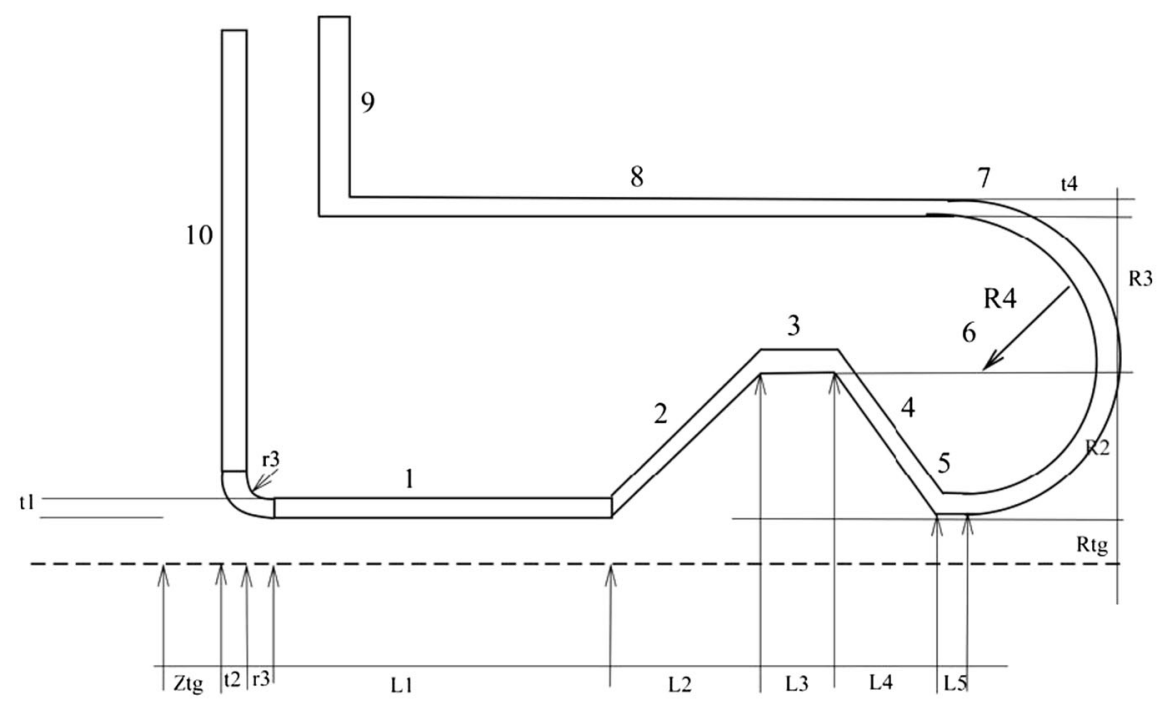

FIG. 18. Horn parameters. 


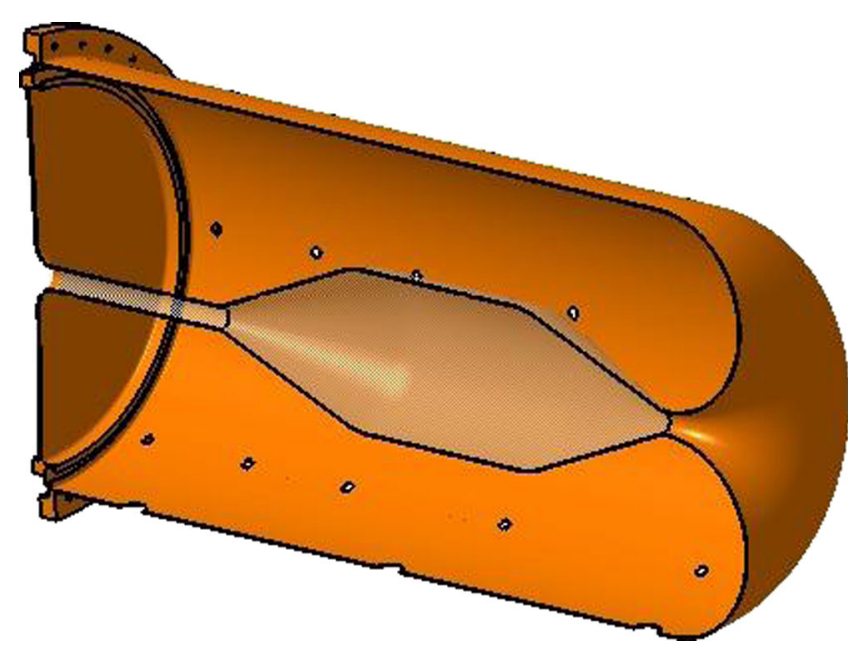

FIG. 19. Cross section of the horn.

for a $2.2 \mathrm{GeV}$ proton beam. An optimization and a redesign has been made in a super beam context $[11,33]$.

New studies of a hadron focusing horn have been done in the context of EUROnu and as a result an optimal closed forward geometry with a separate target has been designed [34,35] — shown in Fig. 18 and Fig. 19 and with geometric parameters reported in Table IV. In summary, a high magnetic field close to the target and small material thickness are desirable to obtain the best meson focusing and minimize multiple scattering and secondary particles' interactions [36].

Given the nominal values of the proton beam power $P=4 \mathrm{MW}$ and the horn current $I_{0}=350 \mathrm{kA}$, a high power density is present inside the target and horn wall conductors. The feasibility of this horn design depends mainly on the temperature and stress level inside the target and horn structure. The stress level needs to be compared to the fatigue strength of the material to give an estimate of the horn lifetime.

The horn will be made of aluminium AL 6061-T6 with 3 (10) $\mathrm{mm}$ thickness for the inner (outer) conductor. The horn is approximately $2.5 \mathrm{~m}$ in length and $1.2 \mathrm{~m}$ in diameter. For the horn assembly, the different parts will be welded at different locations, preferably in the low stress regions. The inner and outer conductor end plates are electrically

TABLE IV. Horn geometric parameters. $z^{t g}$ and $R^{t g}$ correspond to the target upstream face position and the target radius, respectively.

\begin{tabular}{lc}
\hline \hline Parameters & Value $[\mathrm{mm}]$ \\
\hline$L_{1}, L_{2}, L_{3}, L_{4}, L_{5}$ & $589,468,603,475,10.8$ \\
$t_{1}, t_{2}, t_{4}$ & $3,10,10$ \\
$r_{3}$ & 50.8 \\
$R^{t g}$ & 12 \\
$z^{t g}$ & 68 \\
$R_{2}, R_{3}$ & 191,359 \\
\hline \hline
\end{tabular}

insulated with a glass disk of $2.5 \mathrm{~cm}$ thickness. The target with its own cooling system will be inserted inside the central hole of the horn with an inner diameter of $6 \mathrm{~cm}$. Spacers will have to be designed to maintain the target inside the horn. In the following sections we present the electrical, thermal, and mechanical studies of the electromagnetic horn.

\section{Electrical currents and magnetic flux}

An analytic calculation for the toroidal magnetic field in the horn created by the alternate current has been performed. Most of the current inside the inner conductor is flowing in the region $3.1<r<3.3 \mathrm{~cm}$, accordingly to the calculated skin depth. Electrical losses occur in the inner conductor, conical sections, and at the top end of the horn.

\section{Thermal loads and cooling}

In steady state and from the power density distribution, it is possible to calculate the required heat transfer coefficient $h$ to maintain a temperature difference $\Delta T=$ $T_{\text {horn }}-T_{\text {inf }}=40^{\circ} \mathrm{C}$. The cooling efficiency of the system required to maintain a constant temperature inside the horn structure is proportional to the thickness wall $e$ and the power density $q$.

The temperature distribution has been computed for a basic cooling scenario of $\left\{h_{\text {inner }}, h_{\text {horn }}\right\}=$ $\{1,1\} \mathrm{kW} /\left(\mathrm{m}^{2} \mathrm{~K}\right)$ and for an optimized cooling scenario with higher cooling in the hot spot area $\left\{h_{\text {inner }}, h_{\text {corner }}, h_{\text {conv }}\right\}=\{3.8,6.5,0.1\} \mathrm{kW} /\left(\mathrm{m}^{2} \mathrm{~K}\right)$. Here $h_{\text {inner }}, h_{\text {corner }}, h_{\text {conv }}$ are the heat transfer coefficient on the surface of the inner conductor, on the upstream bottom corner (near the target) and on the right side of the upstream bottom plate.

For the uniform cooling, the maximal temperature is $180^{\circ} \mathrm{C}$. When higher cooling is used in the hot spot area, the maximal temperature is $61^{\circ} \mathrm{C}$. The cooling system configuration will have to be chosen according to these $h$ coefficients required to maintain a reasonable maximal temperature around $60^{\circ} \mathrm{C}$. This thermal model shows that the two hot areas are the upstream bottom corner and the downstream part where the inner radius becomes $r=3 \mathrm{~cm}$. These two domains will have to be cooled adequately to avoid any failure.

\section{E. Static mechanical model}

The displacement field has been computed and shows a maximal displacement of $u_{\max }=1.12 \mathrm{~mm}$ occurring in the downstream part of the horn (opposite to the target side).

The maximal stress of $62 \mathrm{MPa}$ occurs in the corner region. This value is well below the aluminium maximal strength but still high in comparison of the Al 6061 T6 fatigue limit for $10^{8}$ cycles. There is also a high stress level in the top inner waist of the horn. This part and segments junctions will require a slight modification to achieve a 


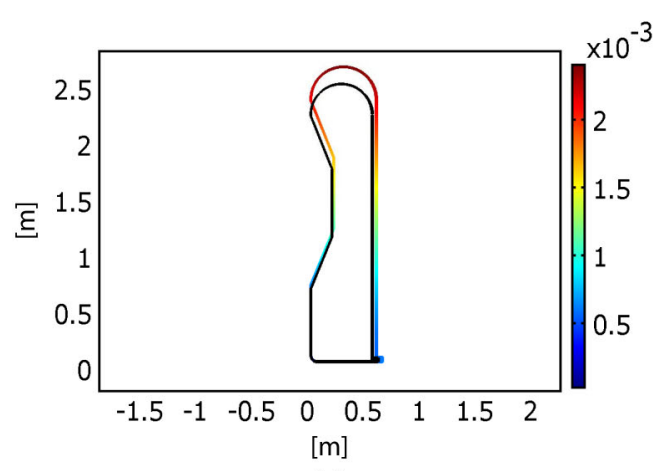

(a)

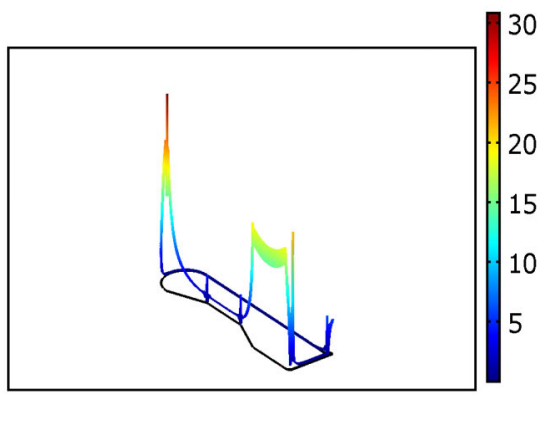

(b)

FIG. 20. Displacement field (in m) (a) and von Mises stress (in MPa) (b) due to thermal dilatation with uniform temperature $T_{\text {horn }}=60^{\circ} \mathrm{C}$.

stress as low as possible below $20 \mathrm{MPa}$ for example. This static thermal stress is due to the thermal gradient due to nonuniform temperature distribution inside the horn.

It is interesting to note that the static stress level can be greatly reduced to $6 \mathrm{MPa}$ if we achieve a uniform temperature. The displacement is about $2.4 \mathrm{~mm}$ in the case of a uniform thermal dilatation with $\Delta T=40^{\circ} \mathrm{C}$.

\section{F. Transient mechanical model}

The transient stress from the magnetic pressure pulse is significant mainly for the inner conductors of the horn with small radius such as the inner conductor parallel to the target and inner waist in the downstream region. The displacement is maximum in the top part of the horn (downstream region, Fig. 20). The displacement due to the magnetic pulse is quite low in comparison to the thermal dilatation. The von Mises stress reaches its maximum value in the upstream corner region. The magnetic pressure pulse contributes for about $20 \mathrm{MPa}$ in the top part of the horn region with $r=3 \mathrm{~cm}$.

The thermal dilatation does not contribute to the radial stress but mainly to the longitudinal stress $S_{z}$ as expected. The thermal static von Mises stress is about 2.5 MPa and the peak stress is $15 \mathrm{MPa}$. Because the inner conductor thickness of $3 \mathrm{~mm}$ is small compared to the inner radius $r_{i}=30 \mathrm{~mm}$ the hoop stress inside the inner conductor is approximately constant with a value of $19 \mathrm{MPa}$.

\section{G. Cooling system}

A system of water jets has been studied for the cooling of the horns' conductors. In particular, the longitudinal configuration of the jets follows the energy density deposition on the conductors. In this system, 6 jets are located at the edges of an hexagon. In total, there are 5 hexagons along the conductor length giving 30 water jets as shown in Fig. 21. The heat sources are electrical resistive losses from pulsed currents and secondary particles generated from the proton beam and target interaction. The heat transfer coefficient depends on the two water phases, the flow rate, the geometry, and the disposition of the nozzles. Assuming an initial inlet temperature and outlet temperature $\left\{T_{i}, T_{\text {outlet }}\right\}=$ $\{20,60\}{ }^{\circ} \mathrm{C}$ and a total power to removed of $Q=22+40=62 \mathrm{~kW}$, the water mass flow rate is $0.37 \mathrm{~kg} / \mathrm{s}$. Hence, assuming ideal heat removal, the minimum water flow rate will be $24 \mathrm{l} / \mathrm{min}$. The final flow rate can be estimated to be in the range of 60-120 1/ min per horn. The flow rate and jets characteristics will be chosen in order to limit the conductor's temperature below a safe limit around $60^{\circ} \mathrm{C}$ and to remain in a single liquid phase cooling regime.

The high heat transfer coefficient required is quite challenging as it demands a high water flow [37]. Further developments are required on the basis of commercial nozzles in order to increase the conventional capacities [38]. Nevertheless, heat transfer coefficient in the range of $10 \mathrm{~kW} /\left(\mathrm{m}^{2} \mathrm{~K}\right)$ can be expected at a flow rate of approximately $4 \mathrm{l} / \mathrm{min}$ with the help of the microchannel technique developed for very-large-scale integration chips at Soreq [39].

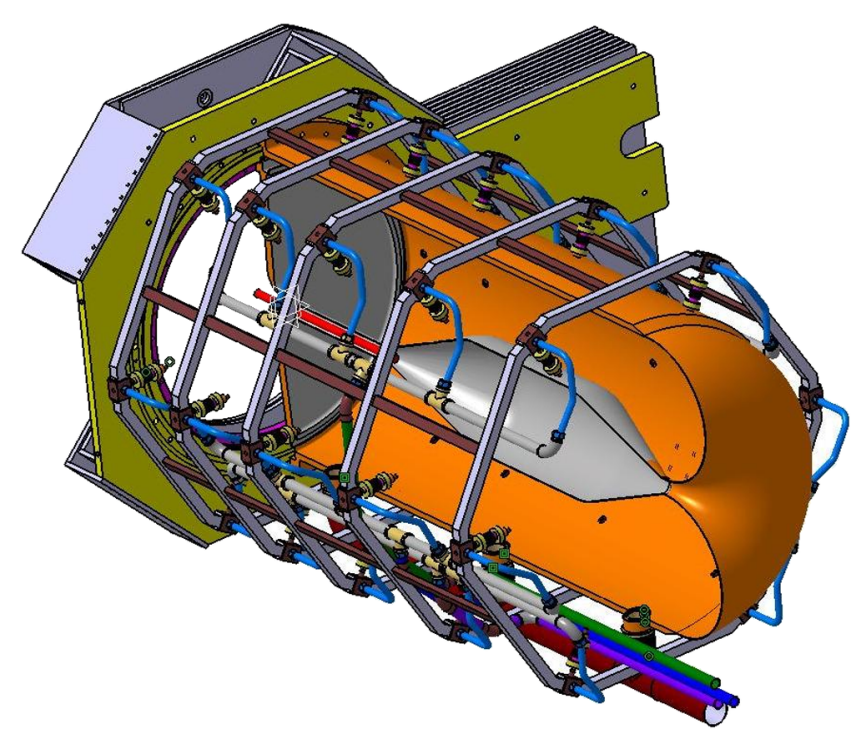

FIG. 21. Horn with strip lines and cooling system. 


\section{H. Modal analysis, natural frequency}

The current pulse circulating inside the horn is of sinusoidal form with a $100 \mu$ s width. The repetition frequency is $12.5 \mathrm{~Hz}$ in normal use with a four-horn system or $16.6 \mathrm{~Hz}$ with three horns running. The first six eigenfrequencies for the current horn geometry are $f=$ $\{63.3,63.7,88.3,138.1,138.2,144.2\} \mathrm{Hz}$ excluding all the pipes and the frame connected to the horn's outer conductor. The first three fundamental modes are related to the inner conductor vibrations; the fourth, fifth, and sixth modes are related to the outer conductor vibrations.

\section{Considerations on fatigue}

The design lifetime of the horn should aim at $10^{9}$ pulses which is about 926 days. The fatigue limit for aluminium alloys depends on the number of cycles. There is no stabilization of the fatigue limit value for the very high number of cycles $\left(N>10^{7}\right)$ [40]. Experimentally obtained stress-to-failure (S-N) curves for wrought aluminium alloy 6082 show that the fatigue limit slowly lowers when increasing the number of cycles within the range of values between $10^{7}$ and $10^{9}$ of cycles [41]. The fatigue data for aluminium alloys can only give a probability of failure for a determined level of stress and number of cycles. In the MiniBooNE horn design [42] the maximum equivalent stress limit is $68 \mathrm{MPa}$ everywhere in the horn to have a $97.5 \%$ confidence level for no failure at $10^{8}$ cycles.

The presence of an initial mean stress due to thermal dilatation reduces the fatigue strength [43]. For sustained cyclic conditions, the material should stay in the elastic regime or in other words any combination of mean stress and alternating stress should not create yielding or plastic deformation.
According to Ref. [43], the fatigue strength limit of dynamic stress is 50 (20) MPa for $10^{9}$ pulses for zero (maximum) mean stress, respectively. For the weld junction with mean stress, a limit of $10 \mathrm{MPa}$ should be used.

For the inner conductor horn, the magnetic pressure pulse creates a peak of the dynamic stress of about $16 \mathrm{MPa}$ of the von Mises equivalent stress. This value is below the $20 \mathrm{MPa}$ limit strength for $10^{8}$ cycles and with mean stress due to thermal dilatation $[44,45]$.

\section{J. Effect of neutrons irradiation}

In the case of a high neutron flux, larger than $6 \times 10^{22} \mathrm{n} / \mathrm{cm}^{2}$, the formation of $\mathrm{He}$ and $\mathrm{H}$ creates cavities and bubbles inside the materials. These defaults lead to a reduction of the mechanical properties of the material [46]. Nevertheless, FLUKA simulations show that the neutrons flux through the horn is much lower than $10^{22} \mathrm{n} / \mathrm{cm}^{2}$, so the material properties should not be degraded by neutron irradiation. The mechanical properties of the aluminium alloy 6061-T6 may change under irradiation by all the secondary particles generated from the proton beam and target interactions and their synergy with the applied stresses [47]. For moderate neutrons' flux the neutrons create the transmutation of ${ }^{27} \mathrm{Al}$ to ${ }^{28} \mathrm{Si}$. This can lead to the formation of a $\mathrm{Mg}_{2} \mathrm{Si}$ precipitate and an increase of the yielding strength (limit of elasticity) and the ultimate tensile strength. Radiation hardening generally decreases the tensile elongation (depending on the alloy). This issue has to be investigated in order to evaluate the impact on the material resilience in the case of fatigue stress.

A first evaluation of the influence of irradiation on the lifetime of the horn inner conductor indicates a strong dependence of the number of cycles to failure on the maximum displacements per atom (dpa) [45,48].

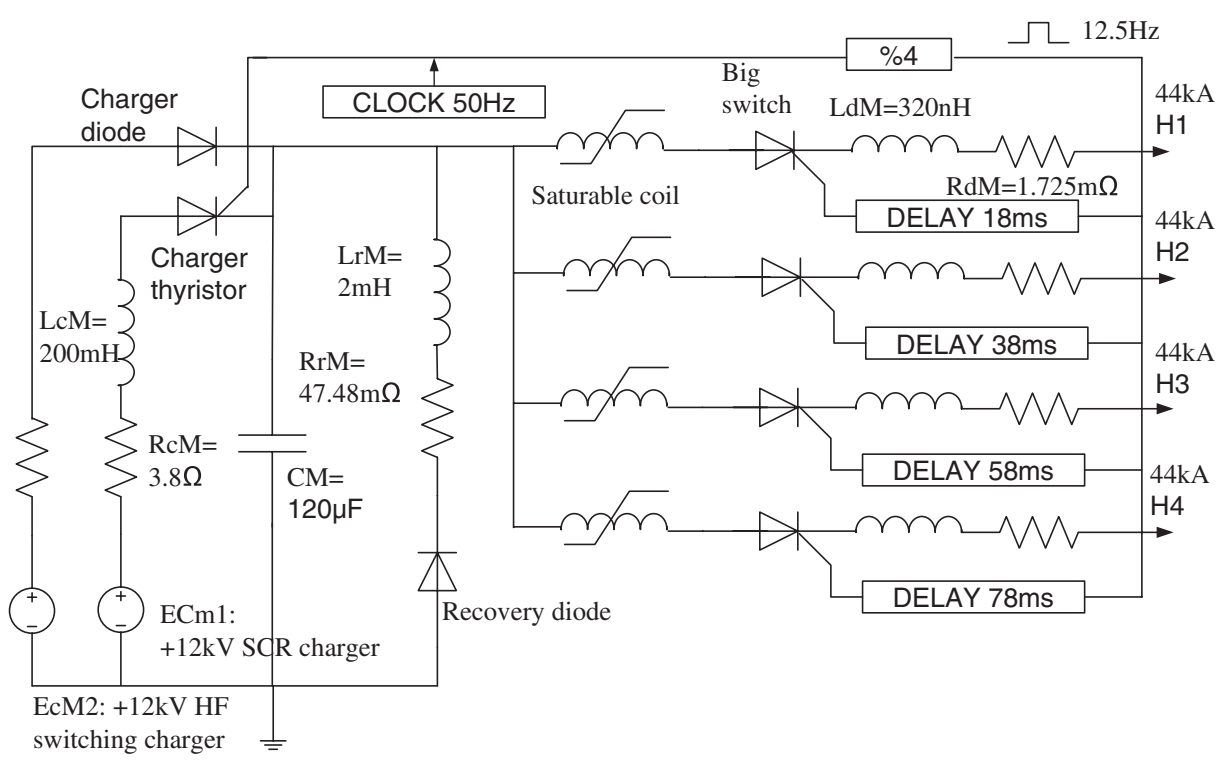

FIG. 22. Diagram of a power supply module. 


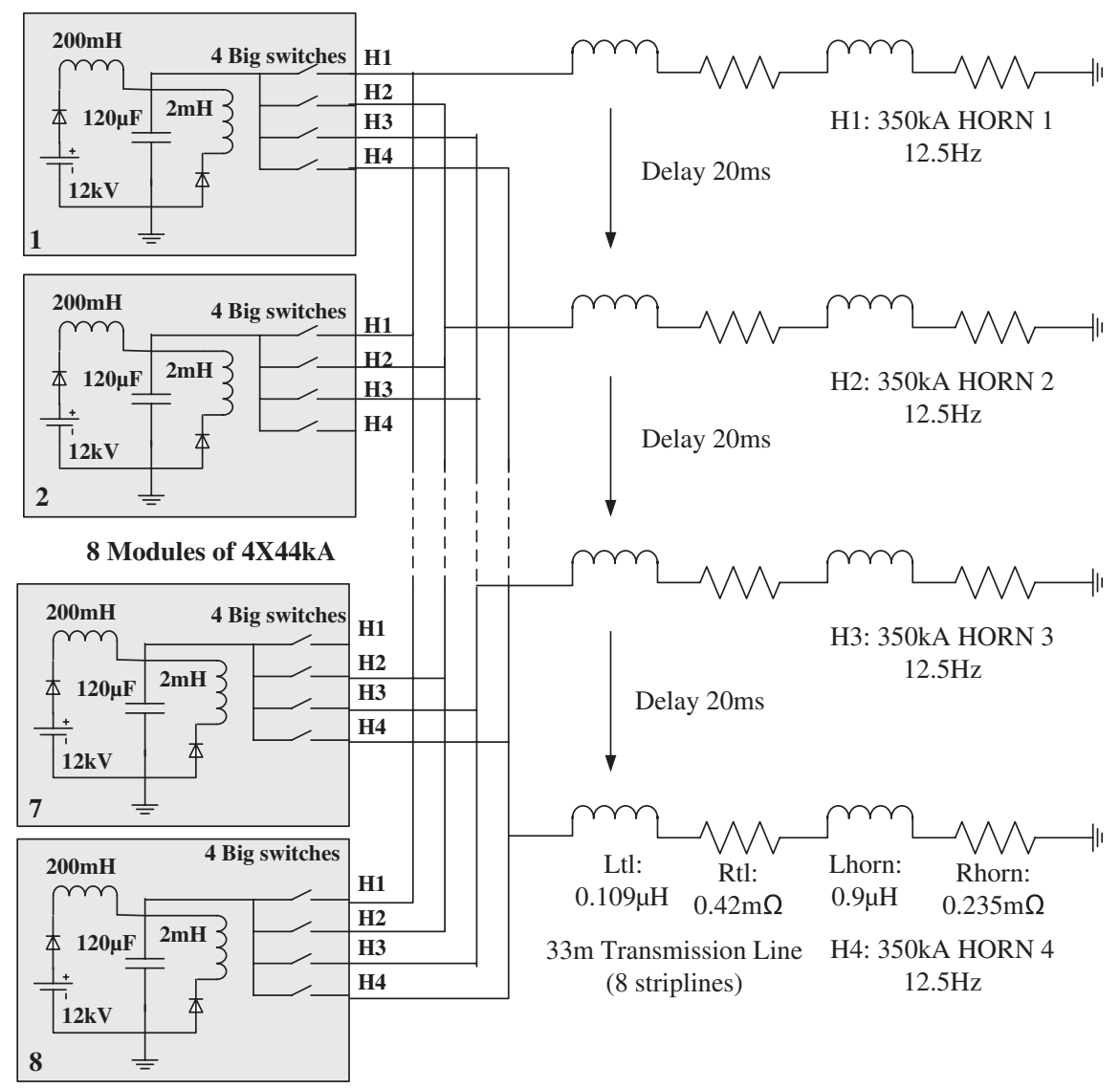

FIG. 23. Modular architecture of the horn's power supply.

A parametric study involving both irradiation induced microdamage (Frenkel pairs, microvoids, microcavities containing $\mathrm{He}$ ) and mechanically induced damage fields (microcracks and microvoids) shows that for the maximum dpa not exceeding $10^{-5}$, the number of cycles to failure reaches more than $10^{5}$. Each higher level of dpa leads to a strong reduction in the number of cycles to failure, following a power law. In particular, a dpa level of 0.1 may already compromise the integrity of the inner conductor. In order to confirm these values and establish the range of safe performance of the horn, further research and development study is necessary. One of the crucial parameters that still remains to be confirmed is the evolution of dpa as a function of time (number of cycles) in the inner conductor part located in direct proximity of the target. Such a study will result in final confirmation of the lifetime of the target horn.

Although multiphysics simulation of the whole system can greatly help the conception of a reliable design, dedicated research and development and testing with a target will be needed in the future to not only validate these studies but also to face the various safety aspects (chemistry of heavy metals, high radiation levels, high voltage, high current, etc.), which would also include the design of a complete remote handling installation for the horn and target maintenance and possible exchange.

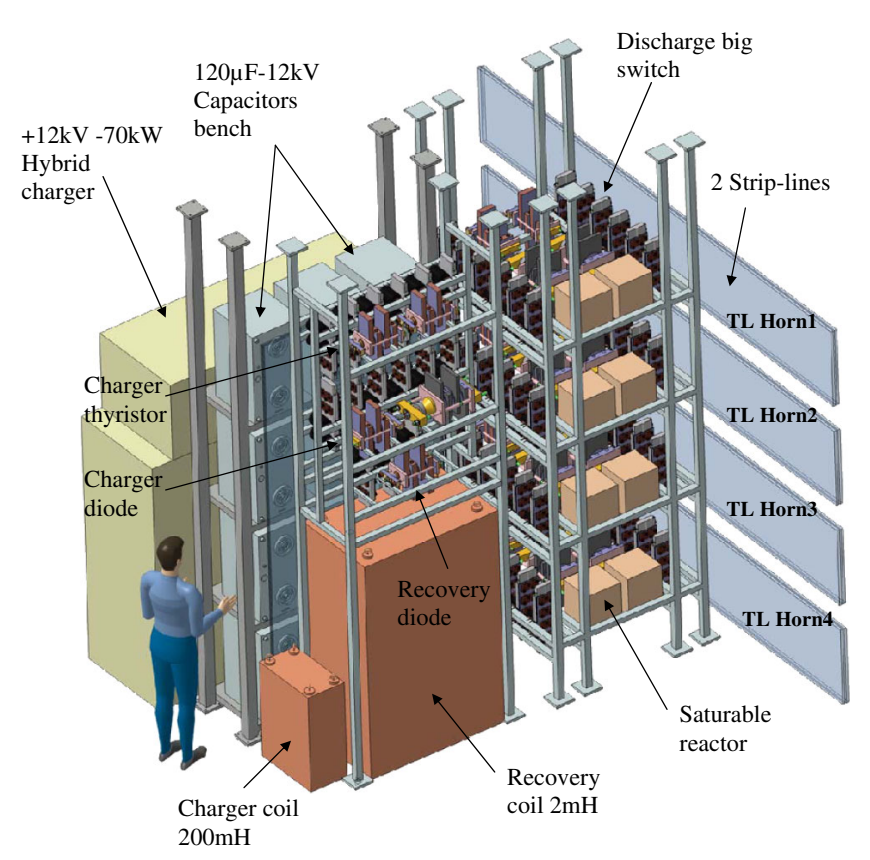

FIG. 24. Artist's view of a power supply unit. 


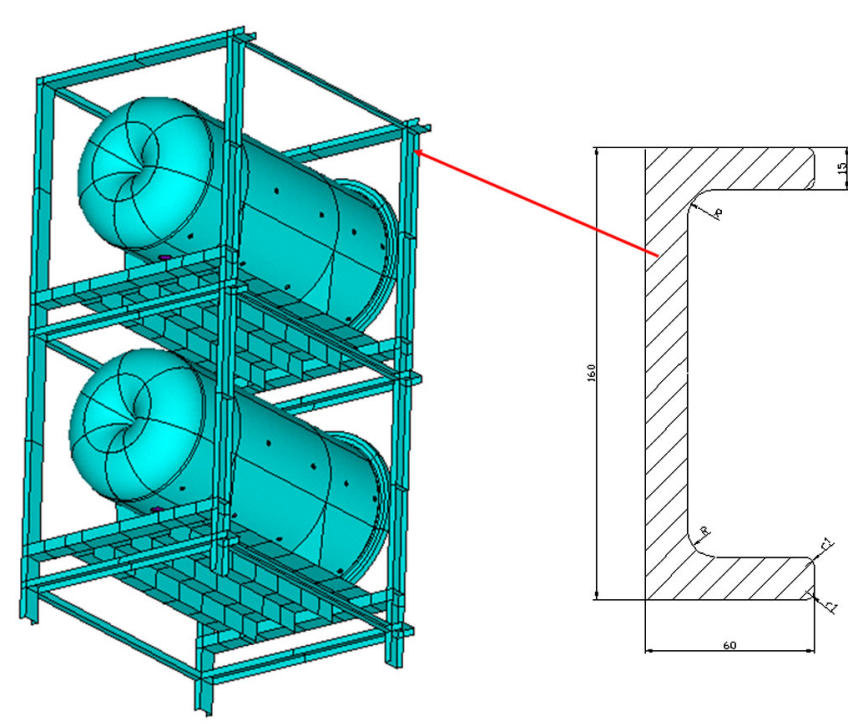

FIG. 25. A symmetric half of the four-horn assembly with detail of the channel section used for the supporting frame.

\section{K. The horn power supply}

We have studied a power supply to provide the halfsinusoidal waveform current to the horn. A capacitor charged at $+12 \mathrm{kV}$ reference voltage will be discharged through a large switch in a horn via a direct coupled design (Fig. 22). A recovery stage allows one to invert rapidly the negative voltage of the capacitor after the discharge, and to limit the charge capacitor current.

We have adopted a modular architecture with eight units (Fig. 23): Two modules are interconnected on a same transmission line based on two strip lines with resistance Rtl $=1.683 \mathrm{~m} \Omega$ and inductance Ltl $=435 \mathrm{nH}$. To limit the energy consumption and therefore the current delivered by the $12 \mathrm{kV}$ capacitor charger, investigations have been done to reduce the resistivity and the inductance by studying a transmission line based on large strip lines of aluminium. It allows one to obtain small resistivity of $51 \mu \Omega / \mathrm{m}$ and inductance $13.2 \mathrm{nH} / \mathrm{m}$ for two plates $(0.6 \mathrm{~m}$ height, $1 \mathrm{~cm}$ width) spaced by $1 \mathrm{~cm}$.

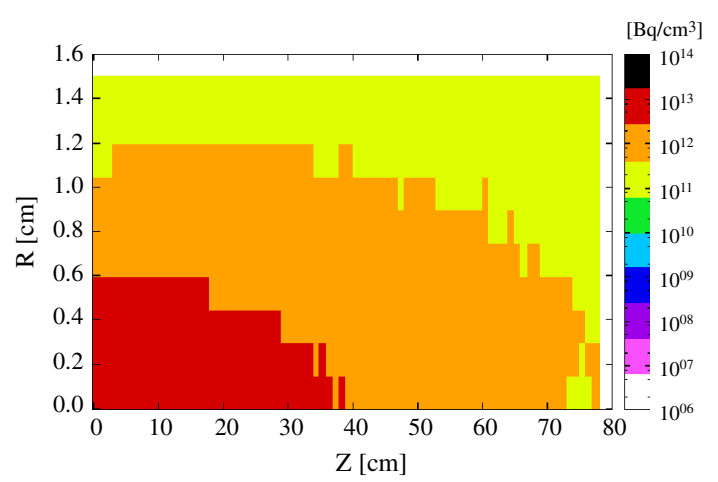

The capacitor charge and recovery circuits operate at $50 \mathrm{~Hz}$; the discharge of current in each horn occurs at a $12.5 \mathrm{~Hz}$ frequency and is delayed by $20 \mathrm{~ms}$ between each horn.

The power delivered by the capacitor charger attains $70.8 \mathrm{~kW} \mathrm{rms} \mathrm{per} \mathrm{module;} \mathrm{that} \mathrm{is} 566 \mathrm{~kW} \mathrm{rms} \mathrm{in} \mathrm{total.} \mathrm{It}$ represents only $3 \%$ of the quantity of the current discharged in the horn, so the recovery energy efficiency is very high $(97 \%)$. An artist's view of one unit is shown in Fig. 24. A more detailed description of this device can be found in [49].

\section{The target and horn support structure}

Following the proposal of the four-horn configuration, a supporting structure for the targets and horns has been proposed. This structure consists of a double-sided frame joined with a system of plates directly supporting the horns (Fig. 25). The thickness of the plate elements and reinforcing ribs are proposed on the base of the numerical optimization results, which was performed for the finite element model of the structure. The minimization of the horns' deflections was the main optimality criteria used in calculations. In parallel the maximum stress in the horns and the supporting structure were monitored. In the next step the dynamic analysis for the optimized supporting structure with the horns was performed in order to check whether the natural and excitation frequencies are well separated. The above described procedure has been performed for two materials used for the supporting system, namely, the aluminium alloy (the same as that used for the horns) and the construction steel. More detailed results can be found in [13].

\section{STUDIES OF ACTIVATION AND SHIELDING}

\section{A. Simulation technique}

A detailed calculation of the target and horn activation has been realized with FLUKA [28,50] version 2011.2.7 in order to study the activation of the target and horn and to determine the thickness of shielding required to comply

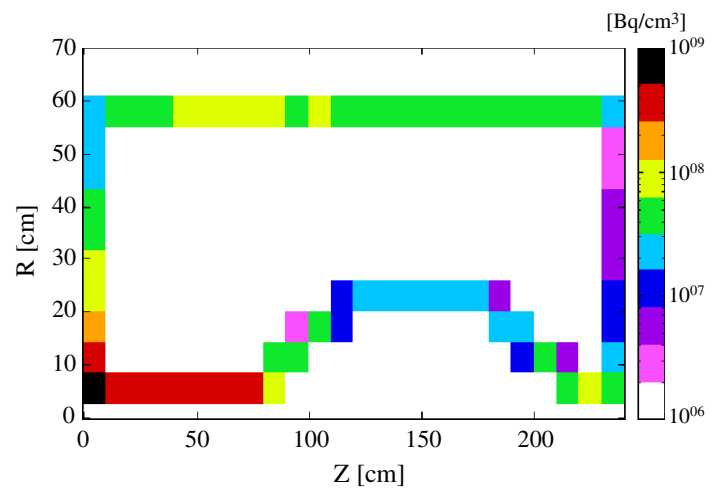

FIG. 26. Spatial distribution of the activation in the target (left) and in the horn (right). 
TABLE V. Energy deposition in $\mathrm{kW}$ for the horns, iron, and concrete around four-horn system for $4 \mathrm{MW}$ proton beam.

\begin{tabular}{lcccc}
\hline \hline Area & Target & Horn & Iron & Concrete \\
\hline$\nu$ beam $(\mathrm{kW})$ & 85 & 32 & 437 & 0.01 \\
anti- $\nu$ beam $(\mathrm{kW})$ & 85 & 32 & 496 & 0.01 \\
\hline \hline
\end{tabular}

with the radiological regulations. The calculations have been done by considering 200 days of irradiation with a $4.5 \mathrm{GeV}$ proton beam of $1 \mathrm{MW}$ intensity impinging a solid target. The packed bed target with titanium spheres chosen as the baseline target option is modeled as a continuous medium with a reduced density of $3 \mathrm{~g} / \mathrm{cm}^{3}$. The results are used as input to the finite-elements calculations for the heat dissipation and the design of the cooling methods for the titanium target, the aluminium horn, and the graphite beam dump.

\section{B. Target and horn studies}

The evolution of the induced activation has been estimated as a function of cooling time for the target and the horn. The value of the specific activity is obtained as a mean value over the total mass of the considered element.

The activation of the target is nonuniform and presents the most active part upstream of the target. The profile of the activation follows the energy deposition inside the target with respect to the beam profile (Fig. 26). After one year of cooling time, the remaining radionuclides contributing to the total activity of the horn $\operatorname{are}^{3} \mathrm{H},{ }^{7} \mathrm{Be}$, ${ }^{10} \mathrm{~B},{ }^{14} \mathrm{C},{ }^{22} \mathrm{Na}$, and ${ }^{26} \mathrm{Al}$ but only the gamma emitters have a significant impact on the radiological aspect especially in the case of ${ }^{7} \mathrm{Be},{ }^{22} \mathrm{Na}$ and the long-lived isotopes ${ }^{26} \mathrm{Al}$.

As in the case of the titanium target, the activation is not uniform inside the horn and presents the most active region in the inner conductor as expected (Fig. 26). Precautions have to be taken in the construction of this part of the inner conductor to prevent cracks due to the amount of radiation (water leaks, etc.).

A simplified simulation has been realized to evaluate the contribution to the ambient dose rate around the target and the horn thanks to the AMB74 option of FLUKA [28,51]. In this study, a two step method has been used to evaluate the contribution of each of the elements [52]. In this simplified model, all the elements contribute to the dose rate at a non-negligible level. The vessel has an important contribution. The concrete has the lowest contribution to the dose rate but the vessel acts as a thin shield in the evaluation. After one year of operation, the contribution of the horn is still high at the level of $1 \mathrm{~Sv} / \mathrm{h}$, which prevents human intervention even by removing the target which is the most active part by 2 orders of magnitude compared to the horn.

\section{Target station shield}

The simulated geometry and the power densities of the surrounding iron and concrete of the four-horn area are presented in Table V. Results are presented for both neutrino and antineutrino beams. A small increase in energy deposition for the antineutrino beam is due to positive pions defocusing: more positive secondary particles are produced due to proton-beam charge. Minimal energy deposition is seen on the concrete after the iron. The iron vessel and the shield will be cooled with water pipes.

\section{Decay tunnel}

The decay tunnel area (Fig. 27) consists of the main iron vessel, where the particles decay and neutrinos are produced, and the concrete surroundings in order to protect the molasse from activation. At the beginning of the decay

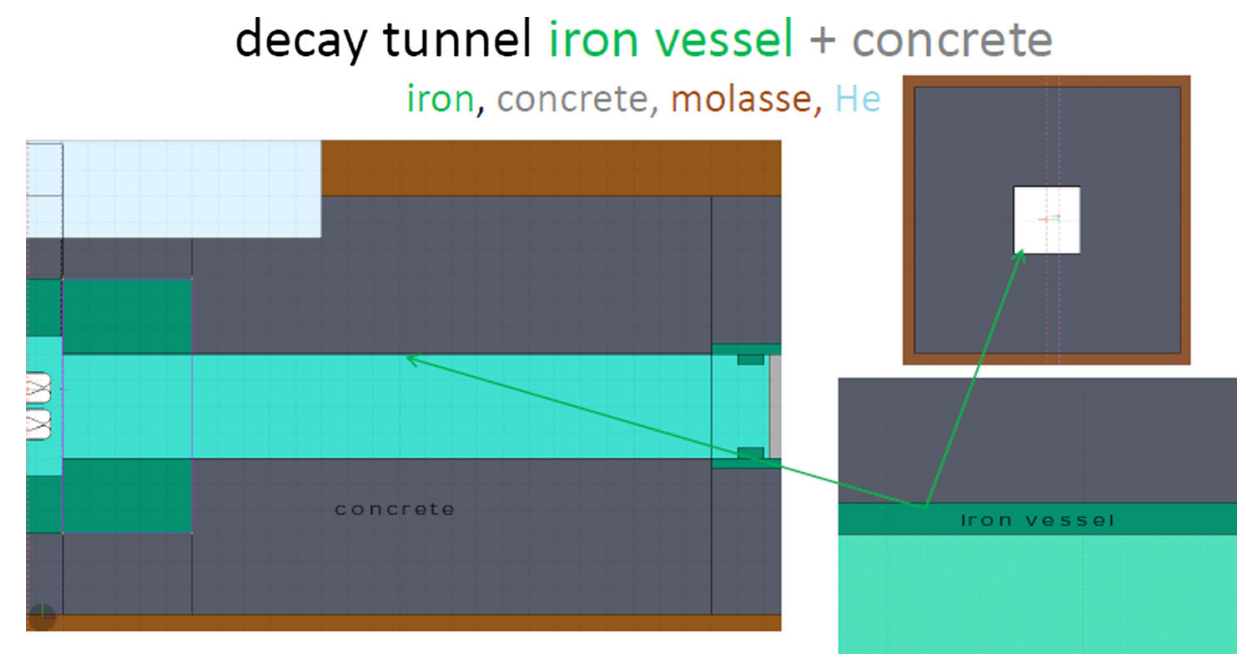

FIG. 27. Decay tunnel layout. 
TABLE VI. Energy deposition in kW for the decay tunnel iron shield, iron vessel, and surrounding concrete.

\begin{tabular}{lccc}
\hline \hline Area & $\begin{array}{c}\text { DT iron } \\
\text { shield }\end{array}$ & $\begin{array}{c}\text { DT iron } \\
\text { vessel }\end{array}$ & $\begin{array}{c}\text { DT surrounding } \\
\text { concrete }\end{array}$ \\
\hline$\nu$ beam $(\mathrm{kW})$ & 610 & 390 & 485 \\
anti- $\nu$ beam $(\mathrm{kW})$ & 775 & 392 & 588 \\
\hline \hline
\end{tabular}

tunnel an upstream iron shield is also foreseen to protect the areas above it like the strip lines. The horn power supply will be built above the start of the decay tunnel.

The energy deposition for the decay-tunnel iron vessel, concrete, and upstream iron collimator are shown in Table VI. The decay tunnel (DT) vessel will be cooled by water pipes.

\section{E. Beam dump}

The beam dump area for the SPL Super Beam follows the design of the T2K [22]. It consists of the main graphite block and several shields (Fig. 28) with the purpose of dumping the remaining hadron particles and finally confining the hadronic energy within the experimental layout.

The energy deposition values are shown in Table VII. The beam dump absorbs all the remaining hadrons so the activation of molasse or any other installation after the beam is prevented. As a result of that, high power dissipation is developed on the dump. Additional studies show that the induced radioactivity in molasse is kept well under CERN's limits $[53,54]$.

\section{F. Shielding investigation}

A first approach for the estimation of the shielding is based on a geometry consisting of a simple iron layer surrounded by concrete. The prompt dose rate can be estimated by using an empirical formula giving the attenuation
TABLE VII. Total energy deposition in $\mathrm{kW}$ for the graphite beam dump and various shields.

\begin{tabular}{|c|c|c|c|c|c|}
\hline Area & Graphite & $\begin{array}{c}\text { Up } \\
\text { shield }\end{array}$ & $\begin{array}{l}\text { Down } \\
\text { shield }\end{array}$ & $\begin{array}{l}\text { Outer } \\
\text { shield }\end{array}$ & Surrounding \\
\hline$\nu$ beam $(\mathrm{kW})$ & 778 & 146 & 19 & 1.6 & 4 \\
\hline anti- $\nu$ beam $(\mathrm{kW})$ & 485 & 128 & 12 & 1 & 3.6 \\
\hline
\end{tabular}

$$
H=\frac{H_{0}(\theta)}{R^{2}} e^{-\frac{t}{\lambda}}
$$

where $H$ is the dose rate after the shield, $R$ the distance between the radiation source (target) and the scoring position, $\theta$ the angle between the proton beam axis and the $R$ (vector) direction, $H_{0}(\theta)$ the source term, and $t$ and $\lambda$ the total thickness and the radiation attenuation length, respectively, of the shield material [55]. If the design of the structure of the shielding element is kept similar to the $\mathrm{T} 2 \mathrm{~K}$, by considering the $2.2 \mathrm{~m}$ of iron, the concrete thickness should reach $3.7 \mathrm{~m}$ to reduce the prompt dose rate to a level of $10 \mu \mathrm{Sv}$.

\section{NEUTRINO FLUXES AND PHYSICS REACH}

\section{A. Physics performances}

This section summarizes the method to optimize the target and horn setup and the main results on the physics performances appearing in [27]. The fluxes and physics performances have been updated for the pebble-bed target configuration described earlier. The neutrino energy spectra are calculated using a probabilistic approach in order to obtain reliable results in a reasonable amount of time using samples of $\sim 10^{6}$ simulated protons. The probability that the neutrino will reach the far detector is calculated at each particle decay yielding neutrinos with analytic formulas $[5,10,12,33,56]$. The probability is then used as a weight

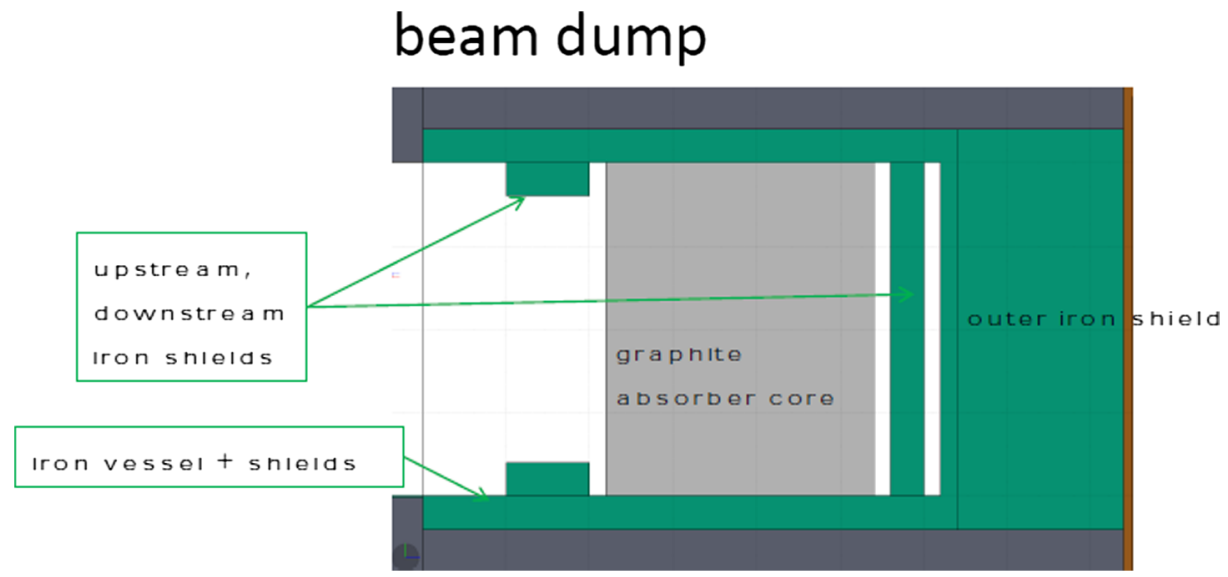

FIG. 28. Beam dump layout used in simulation. Graphite beam dump in grey and several iron shields in green. 

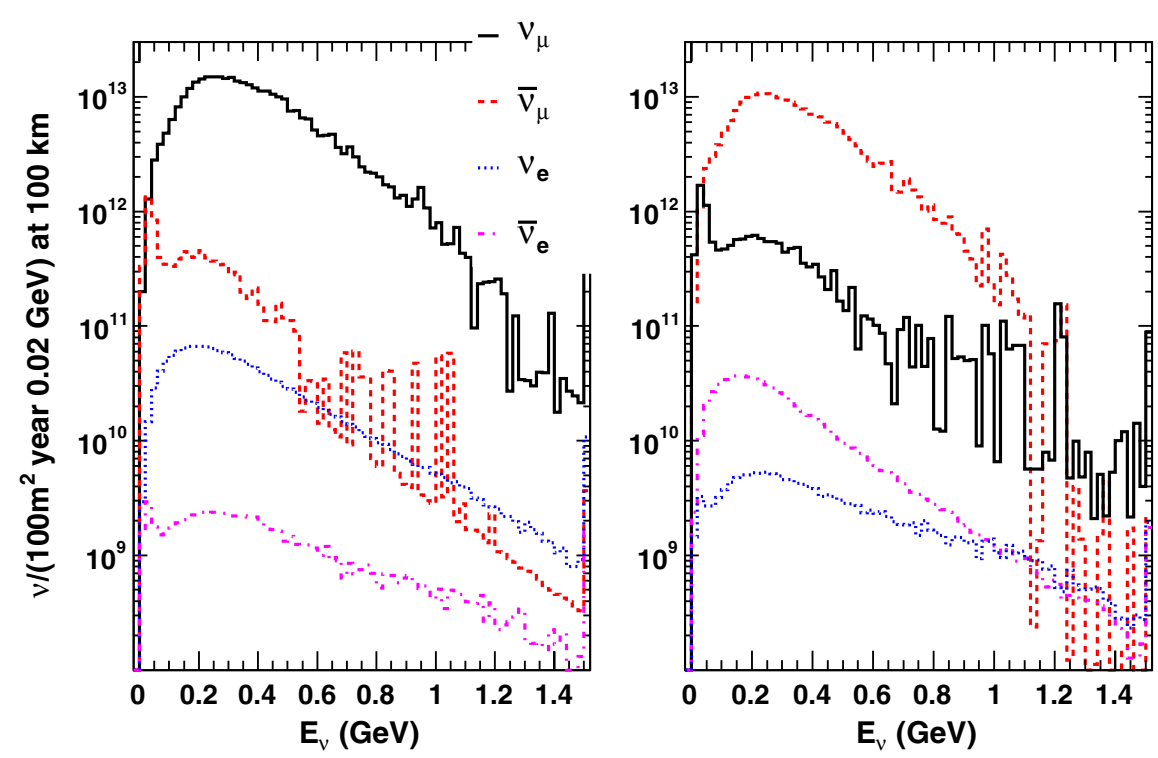

FIG. 29. Nonoscillated neutrino fluxes obtained with the optimized horn and decay tunnel in positive (left) and negative (right) focusing mode.

factor in the calculation of the neutrino energy spectrum. Neutrinos from hadron interactions in the walls of the decay tunnel or in the beam dump are neglected in this approach.

The distribution of the secondaries exiting the target obtained with the FLUKA [28] generator is used as an external input to a GEANT4 [57] simulation derived from a GEANT3 [58] code developed in [10]. The target, the horn with its magnetic field, and the decay tunnel are fully simulated within GEANT4. Alternatively GEANT4 can be used to simulate also the interactions of primary protons in the target; this option was used as cross-check. In order to cross-check and validate the new GEANT4-based software, a comparison has been done with the fluxes obtained with GEANT3. The fluxes obtained in the two frameworks are in good agreement both in terms of normalization and shape [59]. Further cross-checks included the correct implementation of the decay branching ratios, a comparison with an independent code, and a check based on direct scoring of the emitted neutrinos.

The sensitivities for the measurement of the oscillation parameters $\theta_{13}$ and $\delta_{C P}$ are obtained with the help of GLoBES 3.0.14 [60].

TABLE VIII. Integral neutrino flux per year for each flavor at a distance of $100 \mathrm{~km}$ over a surface of $100 \mathrm{~m}^{2}$. The fluxes were obtained with a sample of $10^{7}$ simulated proton-target interactions.

\begin{tabular}{lcccc}
\hline \hline Focusing & $\nu_{\mu}$ & $\bar{\nu}_{\mu}$ & $\nu_{e}$ & $\bar{\nu}_{e}$ \\
\hline Positive & $3.4 \times 10^{14}$ & $9.8 \times 10^{12}$ & $1.6 \times 10^{12}$ & $7.8 \times 10^{10}$ \\
& $96.8 \%$ & $2.8 \%$ & $0.45 \%$ & $0.022 \%$ \\
Negative & $1.6 \times 10^{13}$ & $2.2 \times 10^{14}$ & $1.6 \times 10^{11}$ & $7.0 \times 10^{11}$ \\
& $6.8 \%$ & $92.9 \%$ & $0.07 \%$ & $0.30 \%$ \\
\hline \hline
\end{tabular}

\section{B. Target and horn optimization}

The approach that was followed in the optimization of the forward-closed horn and the decay tunnel uses the final $\sin ^{2} 2 \theta_{13}$ sensitivity. This is a way to maximize the flux at the first oscillation maximum. In this way the final physics performance is used as a guiding principle in the ranking of the configurations under scrutiny. In the evaluation of this quantity a complex set of relevant factors are given as an input: the normalization and shape of each neutrino flavor, the running time in the positive and negative focusing mode, the energy dependence of the cross sections, and the backgrounds in the far detector and its response in terms of efficiency and resolution.

We define the $\delta_{C P}$-dependent $99 \%$ C.L. sensitivity limit as $\lambda_{99}\left(\delta_{C P}\right)$. Averaging on $\delta_{C P}$ and multiplying by $10^{3}$ we introduce

$$
\lambda=\frac{10^{3}}{2 \pi} \int_{0}^{2 \pi} \lambda_{99}\left(\delta_{C P}\right) d \delta_{C P}
$$

This quantity has been used as a practical way of defining with a single number the quality of the focusing system.

The key parameters defining the horn and tunnel geometry are randomly sampled within specified ranges and the correlations with the figure of merit $\lambda$ studied. The parameters of the forward-closed horn and of the decay tunnel were sampled with uniform probability distributions imposing the configuration to be geometrically consistent ("iteration 1"). After studying the correlation of these parameters with the figure of merit, a second iteration was performed with a restriction of the phase spaces around the most promising values. The geometrical parameters obtained with this optimization were reported previously in this article. 

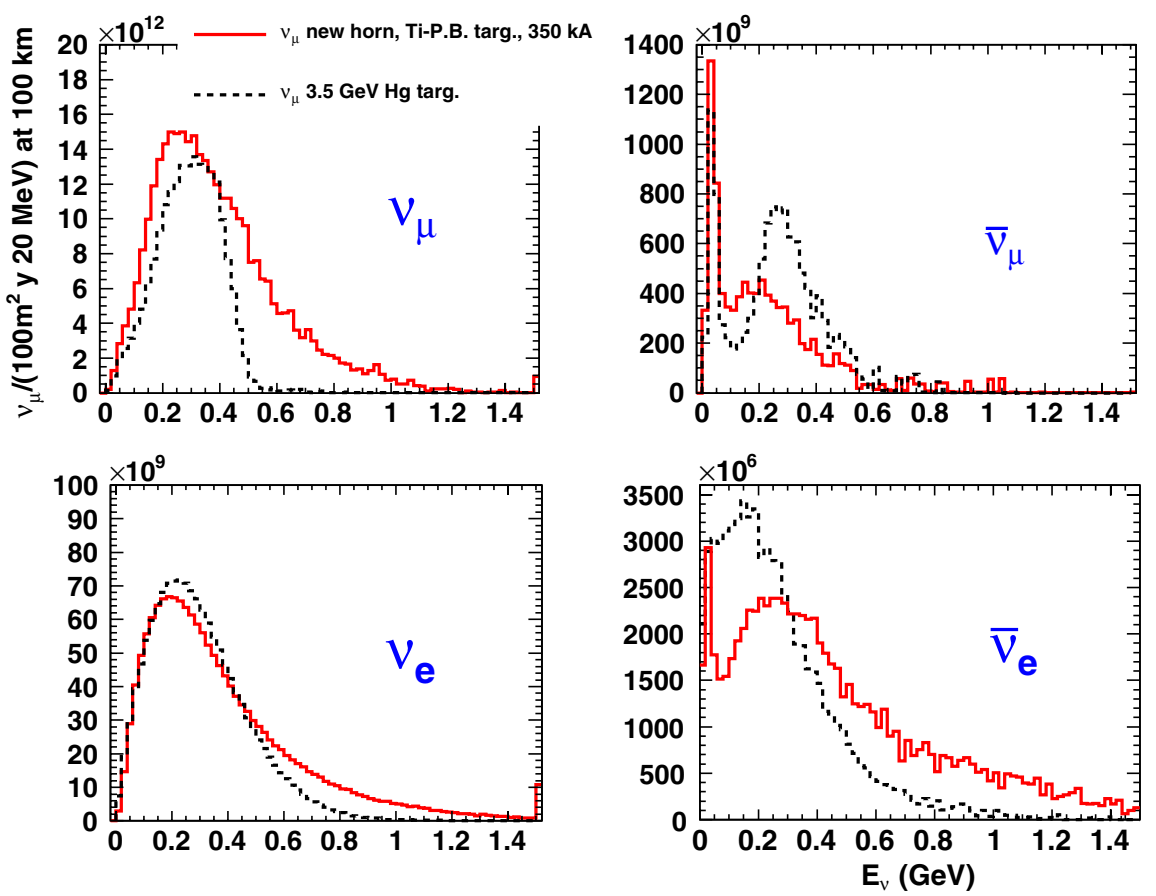

FIG. 30. Comparison of the neutrino fluxes obtained with the new design (continuous line) and the previous one [12] (dotted line).

\section{Beam fluxes}

The nonoscillated $\nu_{\mu}, \bar{\nu}_{\mu}, \nu_{e}, \bar{\nu}_{e}$, and charged conjugate (c.c.) neutrino fluxes are shown in Table VIII and Fig. 29 for positive (left) and negative focusing (right) runs. The nonoscillated $\nu_{\mu}, \nu_{e}$, and charged conjugate (c.c.) neutrino fluxes are shown in Fig. 29 for positive (left) and negative focusing (right) runs. They correspond to $5.6 \times 10^{22}$ protons on target (p.o.t.)/year $\left(4 \mathrm{MW} \times 10^{7} \mathrm{~s}\right.$ at $4.5 \mathrm{GeV}$ ) and are calculated at a reference distance of $100 \mathrm{~km}$ over a surface of $100 \mathrm{~m}^{2}$.

In positive (negative) focusing mode, the $\nu_{e}\left(\bar{\nu}_{e}\right)$ fluxes are dominated by muon decays: $82 \%(90 \%)$. The c.c. fluxes receive instead a large contribution from kaon three-body decays $(81 \%$ and $75 \%$ in positive and negative focusing, respectively) with muon decays from the decay chain of "wrong charge" pions at low energy contributing for the rest. The fluxes are publicly available [61].

The fluxes obtained with the optimized horn (Fig. 30) have been compared to those obtained with the original double conical horn with currents of 300 and $600 \mathrm{kA}$ associated with a mercury target and published in [12]. The $\nu_{\mu}$ and $\nu_{e}$ energy spectra are shifted to higher energies with an increase in statistics particularly around $500 \mathrm{MeV}$. The $\nu_{\mu}$ flux is enhanced also in the proximity of the oscillation maximum at $260 \mathrm{MeV}$ where the $\nu_{e}$ flux is reduced by a similar fraction. The wrong- $C P$ component $\left(\bar{\nu}_{e}, \bar{\nu}_{\mu}\right)$ on the other hand is reduced by more than a factor 2 .

\section{Physics performances}

The $C P$ violation discovery potential at the $3 \sigma$ level is shown in Fig. 31: discovery is possible in the region above the curves. This means that in that region of the true $\left(\sin ^{2} 2 \theta_{13}, \delta_{C P}\right)$ plane a fit done under the $C P$ conserving hypotheses $\left(\delta_{C P}=0, \pi\right)$ gives for both choices a $\Delta \chi^{2}>9$. The limit obtained with the previous setup associated with a mercury target is shown by the

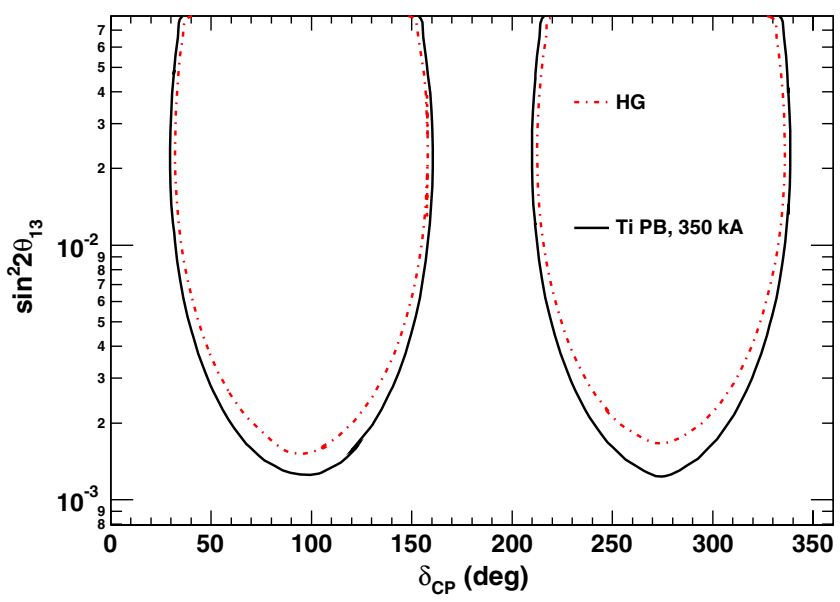

FIG. 31. $C P$ violation discovery potential at $3 \sigma$ level in the $\left(\sin ^{2} 2 \theta_{13}, \delta_{C P}\right)$ plane. The continuous line corresponds to the reference configuration described in this article. The red dasheddotted line corresponds to a notional mercury target considered in previous studies. 
dash-dotted curve while the new limits are represented as a continuous line. The new limits generally improve those obtained with the previous design both for $\theta_{13}$ and $C P$ violation discovery.

\section{CONCLUSIONS}

This study is the first that presents a clear and complete conceptual design for a very challenging facility, capable of delivering a low energy neutrino beam with a $4 \mathrm{MW} 4.5 \mathrm{GeV}$ proton driver. We have presented a novel design for the target, using a split proton beam to divide the power on each device by a factor 4 and a pebble-bed target. The latter allows the coolant to dissipate the heat in a very efficient way, flowing through the innermost part of the target. The structure of the Ti spheres is such that they will stand the static and dynamic stresses. Preliminary calculations show that this target will be able to stand not only $1 \mathrm{MW}$ per device, as originally required, but probably a higher power. This feature makes it a very attractive solution also for other facilities.

The focusing device, a magnetic horn, based on a conventional design, has been optimized for our needs on the basis of a new approach that allows one to study a large parameter space, defined by its geometry, material thickness, current, and the decay tunnel characteristics. This optimization has retained the excellent physics performances while offering a realistic design. Preliminary studies conclude that the lifetime of each device will be sufficient for routine operation with acceptable reliability. A difficult but key component is the power supply, subject to an unusual high repetition rate of $50 \mathrm{~Hz}$ for a peak current of $350 \mathrm{kA}$.

We have studied the most challenging features of the system, starting from the proton beam exiting from the accumulator up to the beam dump. This has required a diverse array of complementary competences and studies that are only briefly summarized here. Our main conclusion is that this project is feasible by adopting the novel approach that we have introduced and developed here. We have fully studied the shielding and activation issues, to comply with existing radiological regulations, and found that the shielding type and thickness, while sizeable, are acceptable. In general, while some of the problems that we had in front of us at the start of the project were particularly challenging, we have found no show stoppers and are confident that this project is feasible.

This study, developed within the context of EUROnu, was limited to the engineering and simulations levels. Some of the devices considered here are novel and would require an extensive phase of research and development to assess the devices' performances and validate with a prototype their use in this context.

\section{ACKNOWLEDGMENTS}

We acknowledge the financial support of the European Community under the European Commission Framework Programme 7 Design Study: EUROnu, Project No. 212372.
[1] J. Beringer et al. (Particle Data Group), Phys. Rev. D 86, 010001 (2012).

[2] O. Brunner et al., Phys. Rev. ST Accel. Beams 12, 070402 (2009).

[3] L. Agostino, M. Buizza-Avanzini, M. Marafini, T. Patzak, A. Tonazzo, M. Dracos, N. Vassilopoulos, D. Duchesneau, M. Mezzetto, and L. Mosca, Phys. Rev. ST Accel. Beams 16, 061001 (2013).

[4] European Commission Framework Program 7 Design Study: EUROnu, Project No. 212372 [http://euronu.org].

[5] M. Donega, Tesi di Laurea, Universita degli Studi di Milano, 2001, http://mdonega.web.cern.ch/mdonega/ lowe/Thesis.pdf.

[6] A.E. Ball, N. Vassilopoulos, A. Blondel, and S.S. Gilardoni, Nucl. Instrum. Methods Phys. Res., Sect. A 472, 650 (2001).

[7] S. S. Gilardoni, Report No. CERN-NUFACT-NOTE-141, 2004.

[8] M. Mezzetto, J. Phys. G 29, 1781 (2003).

[9] S. Gilardoni, G. Grawer, G. Maire, J. M. Maugain, S. Rangod, and F. Voelker, J. Phys. G 29, 1801 (2003).

[10] J.E. Campagne and A. Cazes, Eur. Phys. J. C 45, 643 (2006).

[11] J. E. Campagne, Report No. CERN-NUFACT-NOTE-138, 2004.

[12] J. E. Campagne, M. Maltoni, M. Mezzetto, and T. Schwetz, J. High Energy Phys. 04 (2007) 003.

[13] C. Bobeth et al., WP2 Note 12-01, http://irfu.cea.fr/ Phocea/file.php?class $=$ pisp\&reload $=1351090397 \&$ file $=$ marco.zito/files/87/final-report.pdf.

[14] J. Osborne, A. Kosmicki, and C. Waaijer, Report No. CERN-EDMS 1223890.

[15] C. Densham et al., EUROnu WP2 Note 09-05, http:// heplnv135.pp.rl.ac.uk/joomla/index.php?option=com docman\&task $=$ doc_download\&gid=12\&Itemid $=5$.

[16] C. Bobeth et al., EUROnu WP2 Note 09-11, http:// heplnv135.pp.rl.ac.uk/joomla/index.php?option=com docman\&task=doc_download\&gid=31\&Itemid $=5$.

[17] J. M. Maugain, S. Rangod, and F. Voelker, Report No. CERN-NUFACT-Note- 80.

[18] M. Aiba, Report No. CERN-AB-2008-060-B1, 2008.

[19] E. Benedetto, Report No. CERN-BE-2009-037, Report No. CERN-NUFACT-Note-156, and Proc. NUFACT09.

[20] U. Rohrer, PSI Graphic Transport Framework based on a CERN-SLAC-FERMILAB version by K. L. Brown et al.

[21] ANSYS Academic Research, Release 14.0.

[22] K. Abe et al. (T2K Collaboration), Nucl. Instrum. Methods Phys. Res., Sect. A 659, 106 (2011); K. Abe et al. (T2K Collaboration), Phys. Rev. D 87, 012001 (2013).

[23] J. Hylen, "NuMI Horn Construction and Testing," 4th International Workshop on Neutrino Beams and Instrumentation, Tsukuba, Japan, 2003 (unpublished).

[24] SEC Carbon, Ltd. 1-2-6 Shioe, Amagasaki, Hyogo, Japan 661-0976, http://www.sec-carbon.com/eng/graphite_ specialties.html.

[25] P. Huhr et al., Proceedings of the 46th ICFA Advanced Beam Dynamics Workshop on High-Intensity and HighBrightness Hadron Beams, Morschach, Switzerland, 2010 [http://accelconf.web.cern.ch/AccelConf/HB2010/papers/ tho2c05.pdf]. 
[26] P. Pugnat and S. Sievers, J. Phys. G 29, 1797 (2003).

[27] A. Longhin, Eur. Phys. J. C 71, 1745 (2011).

[28] A. Ferrari, P. R. Sala, A. Fasso, and J. Ranft, Reports No. CERN-2005-10, 2005, No. INFN-TC-05-11, and No. SLAC-R-773; G. Battistoni, F. Cerutti, A. Fassò, A. Ferrari, S. Muraro, J. Ranft, S. Roesler, and P. R. Sala, AIP Conf. Proc. 896, 31 (2007).

[29] ANSYS CFX, release 13.0, Computational Fluid Dynamics, ANSYS, Inc.

[30] S. Ergun, Chem. Eng. Prog. 48, 89 (1952).

[31] E. Achenbach, 7th International Heat Transfer Conference, Munich, Germany, 1982 (unpublished).

[32] S. Kopp, Phys. Rep. 439, 101 (2007).

[33] A. Cazes, Report No. LAL-04-118, 2004.

[34] A. Longhin, EUROnu WP2 Note 10-04, http://heplnv135 .pp.rl.ac.uk/joomla/index.php?option=com_docman\&task $=$ doc_download $\&$ gid=76\&Itemid=5.

[35] C. Bobeth and A. Longhin, EUROnu WP2 Note 10-02, http://heplnv135.pp.rl.ac.uk/joomla/index.php?option=com docman\&task=doc_download\&gid=65\&Itemid=5.

[36] S. van der Meer, Report No. CERN-61-07, 1961.

[37] B. Lepers, EUROnu WP2 Note 6, http://heplnv135.pp.rl.ac .uk/joomla/index.php?option=com_docman\&task=doc_ download\&gid=87\&Itemid $=5$.

[38] M. L. Sentis, A. Ferrari, and S. Rangod, Report No. CERN-OPEN-2006-010, 2006.

[39] H. Hirshfeld, I. Silverman, A. Arenshtam, D. Kijel, and A. Nagler, Nucl. Instrum. Methods Phys. Res., Sect. A 562, 903 (2006).

[40] C. M. Sonsino, International Journal of Fatigue 29, 2246 (2007).

[41] C. Berger, B. Pyttel, and T. Trossmann, International Journal of Fatigue 28, 1640 (2006).

[42] MiniBOONE Collaboration, Technical design report for the MiniBOONE neutrino beam, http://www-boone.fnal .gov/publicpages/8gevtdr2.0.ps.gz.

[43] G. Yahr, in Codes and Standard in a Global Environment, edited by J. Staffiera, Pressure Vessels and Piping Conference, (American Society of Mechanical Engineers, New York, 1993), Vol. 259, p. 43.
[44] P. Cupial and L. Lacny, EUROnu WP2 Note 18, http:// heplnv135.pp.rl.ac.uk/joomla/index.php?option=com docman\&task=doc_download\&gid=170\&Itemid=5.

[45] M.S Kozien and A. Nieslony, EUROnu WP2 Note 19, http://heplnv135.pp.rl.ac.uk/joomla/index.php?option= com_docman\&task=doc_download\&gid=169\&Itemid=5.

[46] J. Campagne, S. Wallon, and B. Kapusta, CERN Report No. NUFACT Note 130.

[47] N. Simos et al., Report No. FERMILAB-CONF-10-480APC, 2012.

[48] A. Ustrzycka and B. Skoczen, EUROnu WP2 Note 17, http://heplnv135.pp.rl.ac.uk/joomla/index.php?option= com_docman\&task=doc_download\&gid=168\&Itemid $=5$.

[49] P. Poussot et al., EUROnu WP2 Note 15, http://heplnv135 .pp.rl.ac.uk/joomla/index.php?option $=$ com_docman\&task $=$ doc_download $\&$ gid $=119 \&$ Itemid $=5$.

[50] V. Vlachoudis, Proceedings of the International Conference on Mathematics, Computational Methods \& Reactor Physics (M\&C 2009), Saratoga Springs, New York, 2009 (American Nuclear Society, LaGrange Park, IL, 2009).

[51] M. Pelliccioni, Radiation Protection Dosimetry 88, 279 (2000).

[52] S. Roesler, 6th Official FLUKA Course, CERN, 2008; S. Roesler (private communication).

[53] CERNSafety Code F, Protection against Ionising Radiations, Revision 1996 (CERN, Geneva, Switzerland, 1996).

[54] S. Agosteo et al., Report No. CERN-TIS-2003-003-RP-CF.

[55] R. H. Thomas and G. R. Stevenson, Radiological Safety Aspects of the Operation of Proton Accelerators, Technical report series No. 283 (International Atomic Energy Agency, Vienna, 1988).

[56] A. Blondel et al., Reports No. CERN-NUFACT-NOTE-53 and No. CERN-NUFACT-NOTE-78.

[57] S. Agostinelli et al., Nucl. Instrum. Methods Phys. Res., Sect. A 506, 250 (2003).

[58] R. Brun et al., GEANT manual, CERN Program Library Long Writeup W5013, 1994.

[59] A. Longhin, AIP Conf. Proc. 1222, 339 (2010).

[60] P. Huber, J. Kopp, M. Lindner, M. Rolinec, and W. Winter, Comput. Phys. Commun. 177, 432 (2007).

[61] The neutrino flux files can be found at http://irfu.cea.fr/en/ Phocea/Pisp/index.php?id=54. 